\title{
Application of a Robust Model Reference Adaptive Control Algorithm to a Nonlinear Automotive Actuator
}

\author{
Alessandro di Gaeta Umberto Montanaro \\ Istituto Motori, National Research Council, Naples, Italy
}

\begin{abstract}
Model reference adaptive control is a viable control method to impose the demanded dynamics on plants whose parameters are affected by large uncertainty. In this paper, we show by means of experiments that robust adaptive methods can effectively face nonlinearities that are common to many automotive electromechanical devices. We consider here, as a representative case study, the control of a strongly nonlinear automotive actuator. The experimental results confirm the effectiveness of the method to cope with unmodeled nonlinear terms and unknown parameters. In addition, the engineering performance indexes computed on experimental data clearly show that the robust adaptive strategy provides better performance compared with those given by a classical model-based control solution with fixed gains.
\end{abstract}

Keywords: Model reference adaptive control, robust adaptive control, nonlinear systems, automotive control, electronic throttle body, electromechanical actuators.

\section{Introduction}

Adaptive control is a versatile control methodology to tame the dynamics of plants affected by large parameter and model uncertainties as the control gains are properly varied, or better adapted, according to the actual system behavior based on a given adaptive mechanism. For these features, adaptive control theory over last three decades has seen significant development, and results are now well assessed and collected in many relevant scientific books as in $[1-5]$. Furthermore, many surveys on the applicability of adaptive schemes to different engineering problems can be found in the technical literature ${ }^{[6-8]}$. Recently, adaptive controllers have been used to tame nonholonomic wheeled mobile manipulators with parameter uncertainties and disturbances ${ }^{[9]}$.

In this paper, we investigate how adaptive controllers can improve closed-loop performance in the case of automotive devices. Indeed, mechatronic automotive systems are often produced in large scale, and their parameters can drastically change from one product to another and over their lifespan. Furthermore, nonlinear unmodeled terms can vary widely as a function of the operating working conditions, making their precise compensation almost impossible (e.g., friction terms that usually affect mechanical plants vary with the temperature and lubrication conditions of the devices.) As a consequence, robust adaptive algorithms are particularly suitable for tackling the complex dynamics of those devices for which there is no precise knowledge of their models or parameters. Nevertheless, full adaptive control schemes are not so common in the automotive industry, and more classical model-based control approaches are still widely used.

To show that advanced adaptive mechanisms can also be particulary useful in this engineering framework, here we consider the case of the adaptive control of a fundamental automotive actuator, i.e., the electronic throttle body (ETB). The control of ETB dynamics represents a perfect example of the control of automotive devices, as the behavior of ETB is affected by many nonlinearities that can alter system performance. Indeed, as often happens, unwanted phenomena, such as stick-slip, hysteresis, hunting limit cycles and impacts, make the control of automotive components a challenging task ${ }^{[10]}$.

Another important issue when dealing with the control of an automotive plant is that the uncertainty affects the system parameters due to manufacturing tolerances, variable operating conditions or mechanical wears. Moreover, simple control strategies are required so that they can be implemented in commercial electronic control units (ECUs) with an acceptable turnaround time.

Although model-based strategies are efficient for controlling automotive mechatronic devices, control performance relies greatly on the knowledge of the plant model. Hence, the robustness with respect to disturbances and parameter variations is a critical issue. Furthermore, a satisfactory parameter identification, which is required by these strategies, can be too time consuming and customized for a single class of product. It is therefore evident that the drawbacks of control strategies with fixed control gains can be overcome with adaptive control laws.

In this work, to face the nonlinearities that typically affect automotive actuators and to impose a preassigned closed-loop behavior, a robust model reference adaptive control (RMRAC) is selected from the adaptive control literature $^{[1]}$. Unlike [10-13], it is an output MRAC strategy. Thus, it does not require the feedback of the entire plant state. This is a remarkable feature when controlling the automotive plants, as only the measurement of their outputs is available to ECUs. Complex state observers can then be avoided, and the industrial requirement of reducing the processing time and the memory needed for the implementation of controllers on an ECU can be met.

The RMRAC algorithm implemented here has other two important advantages with respect to other adaptive methods. First, it allows computation a priori of the max- 
imum tracking error between the plant output and that of the reference model in the presence of disturbances and unmodeled dynamics. Second, it is possible to use a $\sigma$ modification strategy to keep the adaptive gains bounded in a preassigned range without altering the closed-loop stability. Hence, it is particulary suitable for automotive control applications where a high degree of safety is demanded.

The paper is outlined as follows. The RMRAC algorithm proposed in [1] is briefly reviewed in Section 2. The short description of the ETB system and its mathematical model are given in Sections 3 and 4, respectively. The complete design of the adaptive control system for the testbed under consideration is carried out in Section 5 and the maximum tracking error is analytically computed and validated experimentally. Section 6 presents the experimental results over a wide set of realistic maneuvers, showing that this adaptive control method is a promising solution for the control of similar automotive actuators. Section 7 then shows an experimental comparison between the performance provided by the adaptive strategy and that of a more classical modelbased controller in terms of performance indexes that are interesting from an engineering viewpoint.

\section{The RMRAC strategy}

In this section, we briefly review one of the output based RMRAC strategies proposed in [1] for single-input and single-output systems.

For the applicability of the RMRAC it is assumed that the plant dynamics are in the following form:

$$
y=G_{0}(s)\left(1+\Delta_{g}(s)\right)(u+d)
$$

where $u \in \mathbf{R}$ is the control input, $d \in \mathbf{R}$ is an additive disturbance, $\Delta_{g}$ describes possible unknown dynamics, and

$$
G_{0}(s)=K_{p} \frac{N(s)}{D(s)}
$$

is the transfer function of the nominal plant with a known relative degree $n^{\star}$ between the polynomials $N(s)$ and $D(s)$, the coefficients of which are assumed to be completely unknown. Furthermore, it is assumed that the polynomial $N(s)$ is monic and Hurwitz. An upper bound $n$ of the degree $n_{g}$ of $D(s)$ is given, and the sign of the high frequency gain $K_{p}$ is known.

The control objective is to choose $u$ so that all the signals in the closed-loop are bounded and the output $y$ tracks the output $y_{m}$ of a reference model of the form

$$
G_{m}(s)=K_{m} \frac{N_{m}(s)}{D_{m}(s)}
$$

where $N_{m}(s)$ and $D_{m}(s)$ are monic Hurwitz polynomials of degrees $q_{m}$ and $p_{m}$, respectively, with $p_{m} \leqslant n$, and their relative degrees are the same as that of the plant, namely $n^{\star}$.

The control problem formulated above can be solved when the following assumptions are satisfied:

Assumption 1. $\Delta_{g}$ is analytic in $\operatorname{Re}(s) \geqslant-\frac{\delta_{0}}{2}$ for some known $\delta_{0}>0$.

Assumption 2. There exists a strictly proper transfer function $W(s)$ analytic in $\operatorname{Re}(s) \geqslant-\frac{\delta_{0}}{2}$ and such that $W(s) \Delta_{g}(s)$ is strictly proper.
Assumption 3. There exist some constant vectors $\psi_{1}^{\star}$ and $\psi_{2}^{\star} \in \mathbf{R}^{n-1}$, a scalar $\psi_{3} \in \mathbf{R}$, and a Hurwitz polynomial $\Lambda(s)$ of order $n-1$ that can be decomposed as $\Lambda(s)=$ $\Lambda_{0}(s) \cdot N_{m}(s)$ with $\Lambda_{0}(s)$ is a Hurwitz polynomial of order $n-1-q_{m}$, so that

$$
\begin{aligned}
\psi_{1}^{\star \mathrm{T}} \alpha(s) & =\Lambda(s)-N(s) Q(s) \\
\psi_{2}^{\star \mathrm{T}} \alpha(s)+\psi_{3}^{\star} \Lambda(s) & =\frac{Q(s) N(s)-\Lambda_{0}(s) N_{m}(s)}{K_{p}}
\end{aligned}
$$

where $\alpha(s) \triangleq\left[\begin{array}{llllll}s^{n-2} & s^{n-3} & \ldots & s & 1\end{array}\right]^{\mathrm{T}}$ and $Q(s)$ is the quotient of $\frac{\Lambda_{0}(s) D_{m}(s)}{D(s)}$.

Indeed, when these hypotheses are fulfilled, according to [1] (chapter 5, pages 183-189), the reference model dynamics (3) can be imposed in closed-loop by using the following adaptive control law:

$$
u=\psi(t)^{\mathrm{T}} \tau(t)
$$

where

$$
\begin{aligned}
& \tau=\left[\begin{array}{llll}
\tau_{1}^{\mathrm{T}} & \tau_{2}^{\mathrm{T}} & y & r
\end{array}\right]^{\mathrm{T}} \\
& \dot{\tau}_{1}=H \tau_{1}+F u \\
& \dot{\tau}_{2}=H \tau_{2}+F y
\end{aligned}
$$

where $r$ is the input to the reference model (3), $F=$ $\left[\begin{array}{ccccc}1 & 0 & \cdots & 0 & 0\end{array}\right]^{\mathrm{T}} \in \mathbf{R}^{n-1}$, and $H \in \mathbf{R}^{n-1 \times n-1}$ is a Hurwitz matrix in control canonical form so that its characteristic polynomial is $\Lambda(s)$.

The control gains $\psi \in \mathbf{R}^{2 n}$ in (5) are adapted according to the following adaptive mechanisms:

$$
\begin{aligned}
& \dot{\psi}=\varepsilon \Gamma \phi \\
& \varepsilon=-\frac{e+\chi \xi}{m_{s}^{2}}, \quad e=y_{m}-y \\
& \xi=\psi^{\mathrm{T}} \phi+u_{f}, \quad \phi=-G_{m}(s) \tau, \quad u_{f}=G_{m}(s) u \\
& \dot{\chi}=\gamma \varepsilon \xi \\
& m_{s}^{2}=1+n_{d} \\
& \dot{n}_{d}=-\delta_{0} n_{d}+|u|^{2}+|y|^{2}
\end{aligned}
$$

where $\Gamma \in \mathbf{R}^{2 n \times 2 n}$ is a positive symmetric matrix, the parameter $\delta_{0}$ is that in Assumptions 1 and 2, $\gamma$ is a positive constant.

Notice that, different from [14], this algorithm belongs to the class of direct model reference adaptive control, as the plant parameters are not estimated.

In what follows, two fundamental results on the stability of the closed-loop system when the controller (5) is inserted in the control loop are reported.

Theorem 1. Given the plant (2) with $\Delta_{g}=0$ and $d=0$ and the reference model (3). If Assumption 3 is fulfilled then the adaptive control law (5) guarantees global asymptotic convergence of the tracking error, i.e.,

$$
\lim _{t \rightarrow+\infty} e(t)=\lim _{t \rightarrow+\infty} y_{m}(t)-y(t)=0 .
$$

Theorem 2. Given the plant (2) with nonzero plant uncertainties $\Delta_{g}$ and a bounded input disturbance $d$, and 
the reference model (3). If Assumptions 1 to 3 are fulfilled and

$$
c\left(\frac{1}{\alpha_{0}^{2}}+\alpha_{0}^{2 n^{\star}} \Delta_{\infty}^{2}\right)<1, \quad c\left(\frac{1}{\alpha_{0}^{2}}+\alpha_{0}^{2 n^{\star}}\right) \Delta_{2}^{2} \leqslant \frac{\delta}{2}
$$

where

$$
\begin{aligned}
& \Delta_{\infty}=\left\|G_{m}(s) \Delta_{g}(s)\right\|_{\infty \delta_{0}} \\
& \Delta_{2}=\left\|\frac{\Lambda(s)-\psi_{1}^{\star \mathrm{T}} \alpha(s)}{\Lambda(s)} G_{m}(s) \Delta_{g}(s)\right\|_{2 \delta_{0}}
\end{aligned}
$$

and $\delta \in\left(0, \delta_{0}\right)$ is such that $G_{0}^{-1}$ is analytic in $\operatorname{Re}(s) \geqslant-\frac{\delta}{2}$, $\alpha_{0}>\max \left\{1, \frac{\delta_{0}}{2}\right\}$ is an arbitrary constant, and $c \geqslant 0$ denotes a finite constant that can be calculated, then all the signals in the closed-loop plant are bounded and the tracking error satisfies the following inequality for any $T>0$.

$$
\frac{1}{T} \int_{t}^{t+T} e^{2}(\tau) \mathrm{d} \tau \leqslant c\left(\Delta^{2}+d_{0}^{2}\right)+\frac{c}{T}, \quad \forall t \geqslant 0
$$

where $d_{0}$ is an upper bound for $|d|$ and $\Delta^{2}=\frac{1}{\alpha_{0}^{2}}+\Delta_{\infty}^{2}+\Delta_{2}^{2}$.

Remark 1. Assumption 3 guarantees that it is possible to find an ideal static feedback controller so that the dynamics of the plant (2) match those of the reference model (3) when both $\Delta_{g}$ and $d$ are zero. Hence, they represent some matching conditions that are typical for MRAC algorithms. Notice that the knowledge of parameters $\psi_{1}^{\star}, \psi_{2}^{\star}$, $\psi_{3}^{\star}$ is not required for the applicability of the control action (5) since only their existence is demanded. In the case of the ETB, we will show that this hypothesis can be verified without the need to know the plant parameters.

Remark 2. Since the constants $c, \Delta_{2}$ and $\Delta_{\infty}$ depend on unknown transfer functions, plant parameters and $\psi^{\star}$, the conditions for robust stability in Theorem 2 are quite difficult to check a priori for a generic plant. Hence, the importance of the robustness bounds is more qualitative than quantitative. Nevertheless, in the case of the ETB, we will show that when an estimation of the plant parameters is known a first insight on the residual tracking error can be analytically computed. Again we stress that for the applicability of the control algorithm such estimations are not needed; they are used here only to investigate in-depth some closed-loop properties.

Remark 3. In Theorem 2, the norms $\|\mathcal{H}(s)\|_{\infty \delta \delta}$ and $\|\mathcal{H}(s)\|_{2 \delta}$ of a generic proper transfer function $\mathcal{H}(s)$ that is assumed to be analytic in $\operatorname{Re}(s) \geqslant-\frac{\delta}{2}$ are computed as

$$
\begin{aligned}
& \|\mathcal{H}(s)\|_{\infty \delta}=\left\|\mathcal{H}\left(s-\frac{\delta}{2}\right)\right\|_{\infty} \triangleq \sup _{\omega}\left|H\left(\mathrm{j} \omega-\frac{\delta}{2}\right)\right| \\
& \|\mathcal{H}(s)\|_{2 \delta}= \\
& \left\|\mathcal{H}\left(s-\frac{\delta}{2}\right)\right\|_{2} \triangleq\left(\frac{1}{2 \pi} \int_{-\infty}^{+\infty}\left|H\left(\mathrm{j} \omega-\frac{\delta}{2}\right)\right|^{2} \mathrm{~d} \omega\right)^{\frac{1}{2}} .
\end{aligned}
$$

It is possible to prove that there exists the following relation between these norms (see [1] Appendix A, page 331):

$$
\|\mathcal{H}(s)\|_{2 \delta} \leqslant \frac{1}{\sqrt{2 p-\delta}}\|(s+p) \mathcal{H}(s)\|_{\infty \delta} .
$$

for any $p>\frac{\delta}{2} \geqslant 0$.
Remark 4. Theorem 2.2 also provides a bound for the error trajectory computed as

$$
e(t) \leqslant \sqrt{c\left(\Delta^{2}+d_{0}^{2}\right)} \quad \text { almost everywhere (a.e.). }
$$

\section{The electronic throttle body}

The ETB is a mechatronic device used to regulate the air mass flow rate of an internal combustion engine. It is located between the air filter box and the intake manifold. When the throttle plate opens, an airflow sensor detects this change and communicates it to the ECU. As a consequence, the ECU varies the amount of injected fuel to maintain the desired air-fuel ratio ${ }^{[15]}$. The reference signal is the solution of a trade-off between the driver request (acceleration pedal position) and the effective traction possibilities, depending upon drivability, safety, and emission constraints $^{[10,16]}$. The control signal generated by the ECU becomes, by means of an $H$-bridge power converter, the armature voltage of a DC motor. The rotation motion is then transferred from the motor shaft to the plate shaft through a gear system. Fig. 1 shows a schematic of the ETB. Despite its apparent simplicity, the system behavior is affected by many nonlinearities that can dramatically alter its dynamics, such as a piecewise linear restoring torque, friction, impacts and backlash (see [10] for further details). Moreover, the system parameters are often uncertain because of unavoidable manufacturing tolerances, variable operating conditions and mechanical wear ${ }^{[17]}$. On the other hand, the precise control of the plate position of the valve is fundamental not only to regulate the engine torque but also to test on-line fault detection and isolation schemes as those proposed in [18].

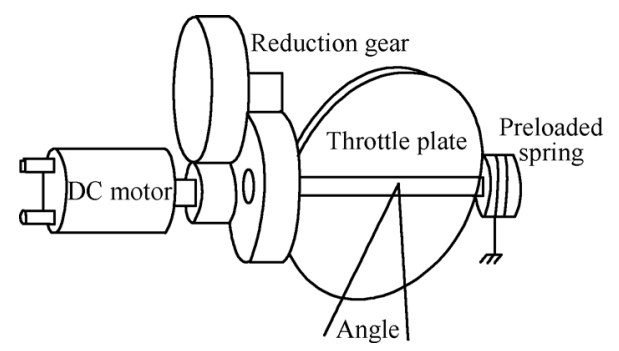

Fig. 1 Schematic diagram of an electronic throttle body

From the control viewpoint, the ETB is a highly nonlinear and uncertain plant, affected by friction due to transmission and nonlinear return spring characteristics. For its features and relevance, the ETB is here chosen as the testbed system to assess performance of adaptive strategies to automotive mechatronic devices. Note that, with the alternative aim of industrializing the device, different control approaches based on well assessed control methodologies have been proposed in the technical automotive literature. To solve the problem, classical controllers, such as those based on a PID structure, are often used ${ }^{[19,20]}$, but these are equipped with some feed-forward model-based action to compensate the nonlinearities acting on the ETB (e.g., [21] and [22]). Further control techniques are based on constrained optimal control ${ }^{[23]}$, robust methods ${ }^{[17]}$, and hybrid approaches ${ }^{[24,25]}$, but again they are based on a good knowledge of the plant dynamics. 
Since the strategy described in Section 2 is adaptive, it can be implemented easily without requiring either the precise characterization of the system nonlinear dynamics or the plant parameters. For the sake of clarity and to emphasize the highly nonlinear nature of the plant and its open-loop dynamics, its mathematical model and some evidence of its dynamical behavior ${ }^{[13]}$ are presented in next section. Furthermore, the system is shown to fit within the class of linear uncertain systems that can be controlled by means of adaptive control law in Section 2.

\section{Mathematical model of the electronic throttle body}

A detailed mathematical model of the plant can be constructed starting from the simple models that describe each part of the system and then considering the interaction between them. The overall model is

$$
\left\{\begin{array}{l}
\frac{\mathrm{d} i}{\mathrm{~d} t}=-\frac{R}{L} i-\frac{K_{v}}{L} \frac{G_{r}}{K} \omega_{t h}+\frac{1}{L} v_{a} \\
\frac{\mathrm{d} \omega_{t h}}{\mathrm{~d} t}=\frac{K K_{t}}{J} G_{r} i-\frac{K}{J} T_{s}\left(\theta_{t h}\right)-\frac{K}{J} T_{f}\left(\omega_{t h}\right) \\
\frac{\mathrm{d} \theta_{t h}}{\mathrm{~d} t}=\omega_{t h}
\end{array}\right.
$$

where $\theta_{t h} \in\left[\theta_{\min }, \theta_{\max }\right]$ (deg) is the plate position, with $\theta_{\min }$ and $\theta_{\max }$ being the minimum and the maximum allowed angles, respectively; $\omega_{t h}(\mathrm{deg} / \mathrm{s})$ is the velocity of the plate; $i$ (A) is the current across the coil of the armature; $v_{a} \in\left[-V_{\mathrm{bat}}, V_{\mathrm{bat}}\right](\mathrm{V})$ is the voltage source across the coil of the armature (with $V_{\text {bat }}$ being the battery voltage); $L(\mathrm{H})$ is the equivalent inductance of the armature coil; $R(\Omega)$ is the equivalent resistance of the armature coil; $K_{v}(\mathrm{Vs} / \mathrm{rad})$ is the velocity constant determined by the flux of the permanent magnets; $K_{t}(\mathrm{~N} \cdot \mathrm{m} / \mathrm{A})$ is the torque constant; $K=\frac{180}{\pi}$ $(\mathrm{deg} / \mathrm{rad}) ; J\left(\mathrm{~kg} \cdot \mathrm{m}^{2}\right)$ is the equivalent moment of inertia; $G_{r}$ is the transmission ratio due to the gear; $T_{s}(\mathrm{~N} \cdot \mathrm{m})$ is the torque due to the presence of the springs, which gives the restoring torque; and $T_{f}(\mathrm{~N} \cdot \mathrm{m})$ represents all friction torques.

The elastic torque, $T_{s}$ in (14), is a piecewise linear function of the admissible angles given by

$$
T_{s}\left(\theta_{t h}\right)= \begin{cases}T_{S_{3}}\left(\theta_{t h}\right), & \text { if } \theta_{t h} \in\left[\theta_{\min } ; \theta_{L H}-\frac{\Delta \theta}{2}\right] \\ 0, & \text { if } \theta_{t h} \in\left[\theta_{L H}-\frac{\Delta \theta}{2} ; \theta_{L H}+\frac{\Delta \theta}{2}\right] \\ T_{S_{1}}\left(\theta_{t h}\right), & \text { if } \theta_{t h} \in\left[\theta_{L H}+\frac{\Delta \theta}{2} ; \theta_{12}\right] \\ T_{S_{2}}\left(\theta_{t h}\right), & \text { if } \theta_{t h} \in\left[\theta_{12} ; \theta_{\max }\right]\end{cases}
$$

being

$$
\begin{aligned}
& T_{S_{1}}\left(\theta_{t h}\right)=K_{s_{1}}\left[\theta_{t h}-\left(\theta_{L H}+\frac{\Delta \theta}{2}\right)\right]+T_{\text {open }} \\
& T_{S_{2}}\left(\theta_{t h}\right)=K_{s_{2}}\left(\theta_{t h}-\theta_{12}\right)+T_{S_{1}}\left(\theta_{12}\right) \\
& T_{S_{3}}\left(\theta_{t h}\right)=-K_{s_{3}}\left[\left(\theta_{L H}-\frac{\Delta \theta}{2}\right)-\theta_{t h}\right]-T_{\text {close }}
\end{aligned}
$$

where $K_{s_{1}}, K_{s_{2}}, K_{s_{3}}(\mathrm{~N} \cdot \mathrm{m} / \mathrm{deg})$ are the stiffness coefficients in each region of interest, $T_{\text {close }}(\mathrm{N} \cdot \mathrm{m})$ is the minimum torque necessary to close the valve, $T_{\text {open }}(\mathrm{N} \cdot \mathrm{m})$ is the minimum torque necessary to open the valve, $\theta_{L H}$ (deg) is known as the limp-home angular position, $\Delta \theta$ (deg) is the clearance between the teeth of the gear, and $\theta_{12}$ (deg) is a further discontinuity point for the slope of the elastic torque $^{[13]}$.

Notice that the elastic torque is provided by a couple of springs that are used for safety reasons. Indeed, if the DC motor fails, it is necessary to ensure that the valve comes back to a default position, i.e., the limp-home position. The limp-home angle corresponds to the supply of a small amount of air into the engine. This guarantees that the valve is not completely closed, thus allowing the driver to "limp" until reaching the nearest car service. As drawbacks, additional discontinuous linearities are introduced in the model around this position, as shown in Figs. 2 (a) and (b).

The model of the friction torque is based on a static Coulomb model modified to include the Stribeck effect as (see Fig. 2 (c))

$$
T_{f}\left(\omega_{t h}\right)=\left[T_{c}+\left(T_{s t}-T_{c}\right) \mathrm{e}^{-\left|\frac{\omega_{t h}}{\omega_{s}}\right|}\right] \operatorname{sign}\left(\omega_{t h}\right)+\beta_{t h} \frac{\omega_{t h}}{K}
$$

where $T_{c}(\mathrm{~N} \cdot \mathrm{m})$ is the Coulomb friction torque, $T_{s t}(\mathrm{~N} \cdot \mathrm{m})$ is the stiction friction torque, $\omega_{s}(\mathrm{deg} / \mathrm{s})$ is the Stribeck velocity, and $\beta_{t h}(\mathrm{~N} \cdot \mathrm{ms} / \mathrm{rad})$ is the equivalent linear damping coefficient $^{[13]}$.

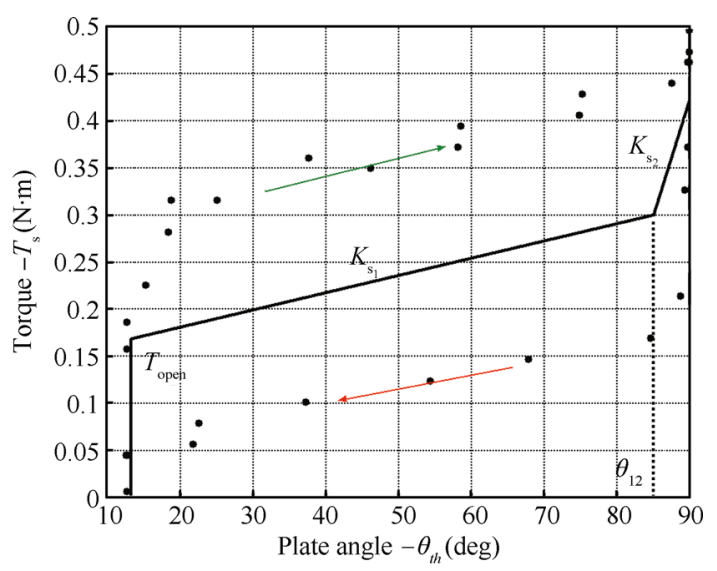

(a)

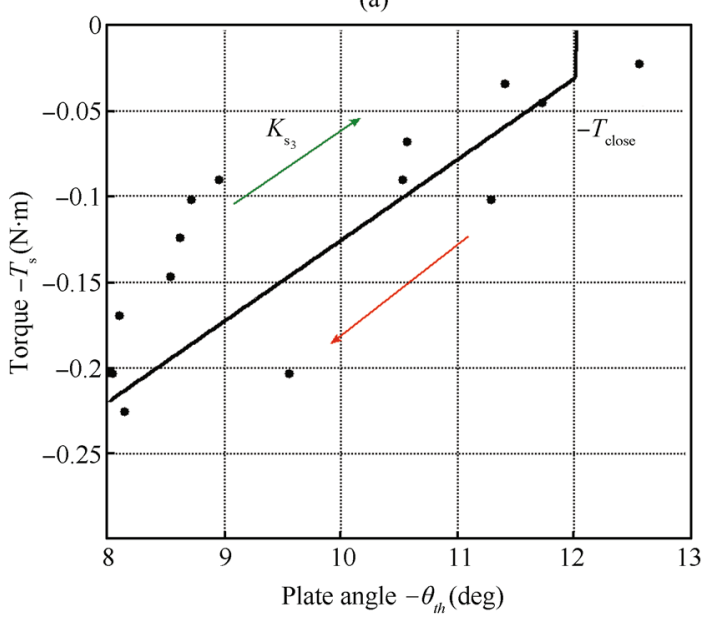

(b) 


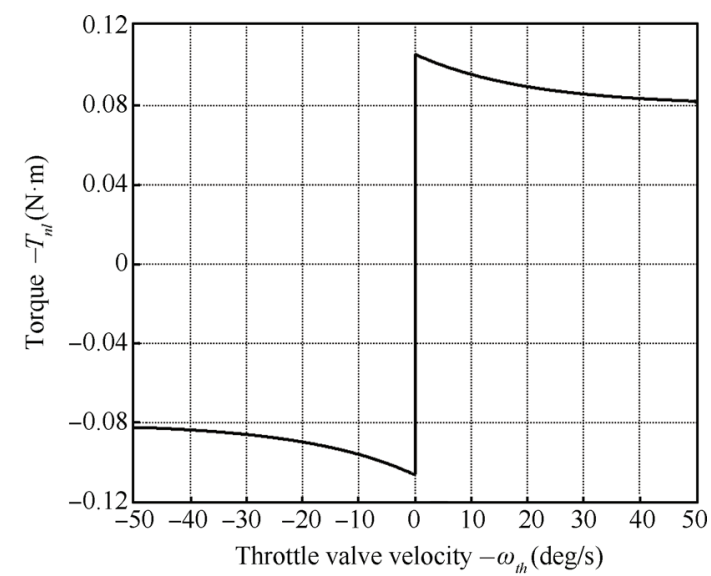

(c)

Fig. 2 Experimental nonlinear characteristics of the plant: (a) Restoring torque when the plate position is greater than the limphome; (b) Restoring torque when the plate position is lower than the limp-home; (c) Nonlinear term of the friction torque

Using the setup described in Appendix, it is possible to capture and confirm experimentally the nonlinear behavior, exhibited by the throttle body. A notable consequence of friction is the presence of stick-slip behavior as shown in Fig. 3 (a). When this kind of unwanted dynamics appear, rigid body elements alternatively stick and slip with respect to each other. Moreover, the combined action of friction and spring torques causes a hysteretic behavior in the system response as confirmed in Fig. 3 (b).

Further details on model derivation, identification, and validation as well as model parameters, can be found in $[10,13]$ and the references therein.

\subsection{Linear approximation of the ETB dy- namics}

In what follows we show that the ETB model (14) can be recast as a linear system ${ }^{[13]}$ with unmodeled dynamics and excited with a nonlinear disturbance as required by the control action as described in Section 2.

To this aim, we rewrite the spring torque as

$$
\begin{aligned}
& T_{s}\left(\theta_{t h}\right)=K_{s_{1}} \theta_{t h}+T_{n l}^{s}\left(\theta_{t h}\right) \\
& T_{n l}^{s}\left(\theta_{t h}\right)= \begin{cases}\Delta T_{S_{3}}\left(\theta_{t h}\right), & \text { if } \theta_{t h} \in\left[\theta_{\min } ; \theta_{L H}-\frac{\Delta \theta}{2}\right] \\
\Delta T_{S_{4}}\left(\theta_{t h}\right), & \text { if } \theta_{t h} \in\left[\theta_{L H}-\frac{\Delta \theta}{2} ; \theta_{L H}+\frac{\Delta \theta}{2}\right] \\
\Delta T_{S_{1}}\left(\theta_{t h}\right), & \text { if } \theta_{t h} \in\left[\theta_{L H}+\frac{\Delta \theta}{2} ; \theta_{12}\right] \\
\Delta T_{S_{2}}\left(\theta_{t h}\right), & \text { if } \theta_{t h} \in\left[\theta_{12} ; \theta_{\max }\right]\end{cases}
\end{aligned}
$$

where

$$
\begin{aligned}
& \Delta T_{S_{1}}\left(\theta_{t h}\right)=-K_{s_{1}}\left(\theta_{L H}+\frac{\Delta \theta}{2}\right)+T_{\text {open }} \\
& \Delta T_{S_{2}}\left(\theta_{t h}\right)=\left(K_{s_{2}}-K_{s_{1}}\right)\left(\theta_{t h}-\theta_{12}\right)+\Delta T_{S_{1}}\left(\theta_{t h}\right) \\
& \Delta T_{S_{3}}\left(\theta_{t h}\right)=\left(K_{s_{3}}-K_{s_{1}}\right) \theta_{t h}-K_{s_{3}}\left(\theta_{L H}-\frac{\Delta \theta}{2}\right)-T_{\text {close }} \\
& \Delta T_{S_{4}}\left(\theta_{t h}\right)=-K_{s_{1}} \theta_{t h}
\end{aligned}
$$

and the friction torque is

$$
T_{f}\left(\omega_{t h}\right)=\frac{\beta_{t h}}{K} \omega_{t h}+T_{n l}^{f}\left(\omega_{t h}\right)
$$

where

$$
T_{n l}^{f}\left(\omega_{t h}\right)=\left[T_{c}+\left(T_{s t}-T_{c}\right) \mathrm{e}^{-\left|\frac{\omega_{t h}}{\omega_{s}}\right|}\right] \operatorname{sign}\left(\omega_{t h}\right) .
$$

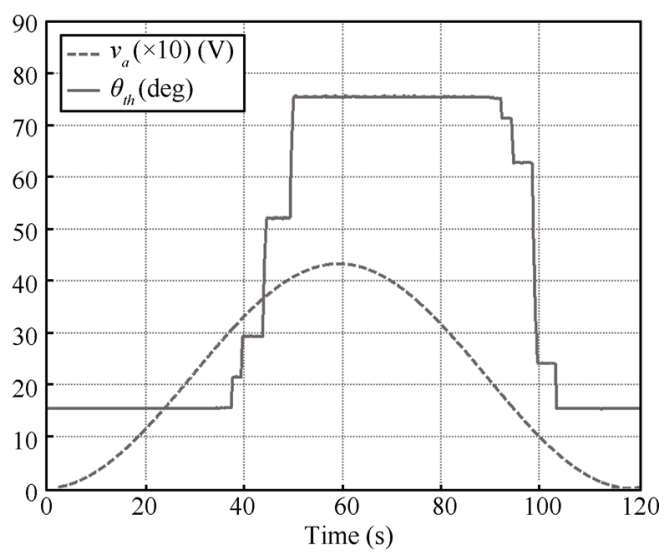

(a)

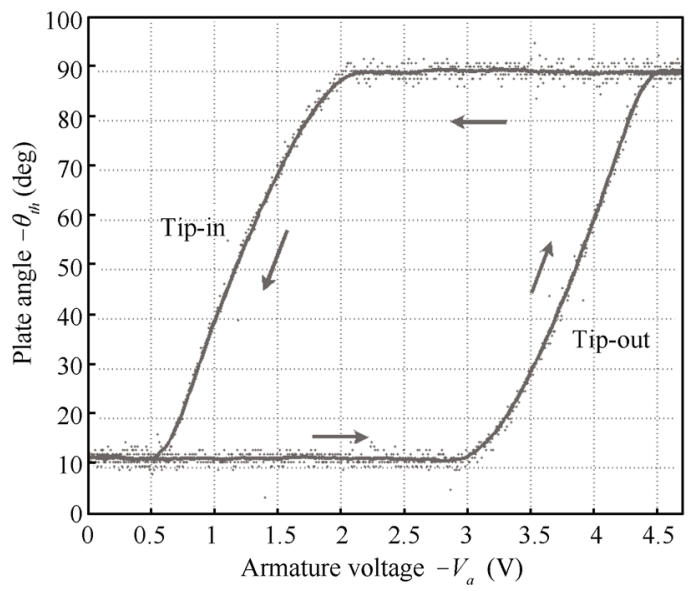

(b)

Fig. 3 Experimental results. (a) Stick and slip phenomena: armature voltage (dashed line) and plate position (solid line). Notice that, to compare the time history of both the variables on the same plot, the armature voltage is multiplied by 10. (b) Experimental hysteresis phenomenon: experimental data from the plant (dotted line) and average data (solid line)

Substituting expressions (18) and (21) in the model (14), we get

$$
\begin{aligned}
\dot{x}= & {\left[\begin{array}{ccc}
-\frac{R}{L} & 0 & -\frac{K_{v}}{L} \frac{G_{r}}{K} \\
0 & 0 & 1 \\
\frac{K K_{t}}{J} G_{r} & -\frac{K K_{s 1}}{J} & -\frac{\beta_{t h}}{J}
\end{array}\right] x+} \\
& {\left[\begin{array}{cc}
\frac{1}{L} & 0 \\
0 & 0 \\
0 & -\frac{K}{J}
\end{array}\right]\left[\begin{array}{l}
u \\
\eta
\end{array}\right] }
\end{aligned}
$$

where $x=\left[\begin{array}{lll}i & \theta_{t h} & \omega_{t h}\end{array}\right]^{\mathrm{T}}$ is the system state, $u=v_{a}$ is the control input, and $\eta(t)=T_{n l}^{s}\left(\theta_{t h}(t)\right)+T_{n l}^{f}\left(\omega_{t h}(t)\right)$ is 
the nonlinear disturbance acting on the linear part of the plant.

The transfer function between $u$ and $y$, say $G_{u y}(s)$, and the transfer function between $\eta$ and $y$, say $G_{\eta y}(s)$, are

$$
G_{u y}(s)=\frac{K K_{t} G_{r}}{J L} \frac{1}{\Phi(s)}, \quad G_{\eta y}(s)=-\frac{K}{J} \frac{\left(s+\frac{R}{L}\right)}{\Phi(s)}
$$

with $\Phi(s)$ being the characteristic polynomial of system (23).

In the case of the ETB, the pole due to the electrical part of the DC motor is at high frequency, that is, it is located around $-\frac{R}{L}$ (electrical pole of the electrical part of the system without the coupling with the mechanical dynamics of the DC motor). With this approximation $\Phi(s)$ can be decomposed as

$$
\Phi(s)=\left(s+\frac{R}{L}\right)\left[s^{2}+\frac{1}{J}\left(\beta_{t h}+\frac{K K_{t}}{R} G_{r}^{2}\right) s+\frac{K K_{s_{1}}}{J}\right] .
$$

Substituting (25) in (24) and after some algebraic manipulations, the input-output model of the system (23) in the Laplace domain has the same structure of the system (2) where

$$
\begin{aligned}
& K_{p}=\frac{K K_{t} G_{r}}{J R}, \quad N(s)=1 \\
& D(s)=\left[s^{2}+\frac{1}{J}\left(\beta_{t h}+\frac{K K_{t}}{R} G_{r}^{2}\right) s+\frac{K K_{s_{1}}}{J}\right] \\
& \Delta_{g}(s)=-\frac{s}{s+\frac{R}{L}}, \quad d(t)=-\frac{R}{K_{t} G_{r}}\left(\frac{L}{R} \dot{\eta}+\eta\right) .
\end{aligned}
$$

\section{Design of the RMRAC strategy for the ETB system}

When a rough plant model in the form (1) is available, the design of the RMRAC requires the following steps:

1) Select a reference model so that certain control specification are satisfied.

2) Verify that Assumptions 1-3 hold with a proper choice of the parameters $\delta_{0}, \psi_{1}, \psi_{2}$ and $\psi_{3}$.

3) When it is possible, compute the residual error (10) so that an estimation of the tracking performance is available before the controller runs.

4) Discretize the control action (5) for its implementation via digital systems and tune the control parameters.

\subsection{Control specifications and reference model}

As always happens when designing MRAC systems, the reference model is derived according to some control objectives. Here we set the control specifications in agreement with automotive requirements ${ }^{[10]}$. More precisely:

1) The settling time is required to be $130 \mathrm{~ms}$ for a valve opening (notice that the settling time is defined as the minimum time after which the throttle plate angle remains within $5 \%$ of its steady-state value).

2) No overshoot should be present in the step response (furthermore, the throttle plate should never hit the mechanical end-stroke).
To meet these control objectives, we select a second order linear time invariant (LTI) system with unitary gain as reference model. This model takes the following form:

$$
G_{m}(s)=\frac{K_{m}}{s^{2}+a_{1} s+a_{2}}
$$

where $K_{m}=a_{2}$.

The poles of the system (27) are chosen so that the reference dynamics track a step reference variation of the setpoint with the preassigned settling time and without overshoots.

\subsection{Fulfillment of Assumptions 1-3}

In the case of the ETB, it is possible to assume that the electrical pole, i.e., $\bar{p}=-\frac{R}{L}$, included in model uncertainty $\Delta_{g}(s)$ in (26c), is at high frequency. And assuming feasible variations of both the electrical inductance and resistance, we have $|\bar{p}|>100 \mathrm{rad} / \mathrm{s}$. Consequently, Assumption 1 is fulfilled selecting $\delta_{0}=200 \mathrm{rad} / \mathrm{s}$. According to this choice of $\delta_{0}$, Assumption 2 is also verified by choosing as $W(s)$ a generic first-order low-pass filter with bandwidth greater than $\frac{\delta_{0}}{2}$.

Note that the reference model is the second-order LTI system in $(27)$, and that in this case $\alpha(s)=1$, after some algebraic manipulations, the matching conditions (4) of Assumption 3 are also satisfied with

$\psi_{1}^{\star}=\frac{1}{J}\left(\beta_{t h}+\frac{K K_{t}}{R} G_{r}^{2}\right)-a_{1}, \quad \psi_{2}^{\star}=-\frac{1}{K_{p}} \psi_{1}^{\star}, \quad \psi_{3}^{\star}=0$.

Note that when both the plant and the reference model are second-order systems without zeros, $\Lambda(s)=\Lambda_{0}(s)=s+\lambda_{0}$.

As it is apparent from (28), it is always possible to verify Assumption 3 since (4) is solvable even if the plant parameters are completely unknown. Here we stress again that the precise value of the parameters $\psi_{i}, i=1,2,3$, is not required for the application of the RMRAC algorithm.

\subsection{Estimation of the residual error}

In what follows an estimation for (13) is derived assuming as plant parameters those given in [10].

The $d_{0}$-term in (10) is computed as

$$
d_{0}=\frac{R}{K_{t} G_{r}}\left(\eta_{m}+\frac{L}{R} \dot{\eta}_{m}\right)
$$

where

$$
\begin{aligned}
\eta_{m}= & \max _{\theta_{t h} \in\left[\theta_{\min }, \theta_{\max }\right]}\left|T_{n l}^{s}\left(\theta_{t h}\right)\right|+\max _{\omega_{t h} \in\left[0, \omega_{\max }\right]}\left|T_{n l}^{f}\left(\omega_{t h}\right)\right| \\
\dot{\eta}_{m}= & \max _{\theta_{t h} \in\left[\theta_{\min }, \theta_{\max }\right]}\left\{\left|\frac{\partial T_{n l}^{s}}{\partial \theta_{t h}}\right|\right\} \omega_{\max }+ \\
& \max _{\omega_{t h} \in\left[0, \omega_{\max }\right]}\left\{\left|\frac{\partial T_{n l}^{f}}{\partial \omega_{t h}}\right|\right\} \dot{\omega}_{\max }
\end{aligned}
$$

where $\omega_{\max }=1200 \mathrm{deg} / \mathrm{s}$ and $\dot{\omega}_{\max }=2000 \mathrm{deg} / \mathrm{s}^{2}$ are the maximum plate velocity and acceleration, respectively, both computed from the model described in Section 4 when the input armature voltage is a step function from zero to the battery voltage. Note that the derivatives of the nonlinear functions have been computed on intervals where their 
gradients exist. This is possible since the bound computed according to (13) is valid almost everywhere (i.e., over intervals with Lebesgue measure different from zero).

The $\Delta_{\infty}$-norm is evaluated considering the frequency response of the transfer function $G_{m}(s) \Delta_{g}(s)$ when the electrical pole of $\bar{p}$ is computed with the nominal plant parameters and $G_{m}(s)$ is that in (27). Finally, the $\Delta_{2}$-norm is evaluated according to (12) with $p=\lambda_{0}$, i.e.,

$$
\Delta_{2}=\frac{1}{\sqrt{2 \lambda_{0}-\delta_{0}}}\left\|\left(s+\lambda_{0}-\psi_{1}^{\star}\right) G_{m}(s) \Delta_{g}(s)\right\|_{\infty \delta_{0}}
$$

with $\psi_{1}^{\star}$ being computed taking into account the nominal plant parameters.

When $\lambda_{0}$ is chosen to be 150 , both the inequalities in Theorem 2.2 are satisfied selecting $\alpha_{0}=120$. Furthermore, we note that $G_{0}^{-1}$ is analytic in the entire complex plane because it is a polynomial.

The resulting approximation of the maximum tracking error is about $9 \mathrm{deg}$. As it will be shown in Section 6, this upper bound is quite good to predict the residual error for time varying reference signals, but it is very conservative in the case of reference set points as the experimental tracking error is always below $0.5 \mathrm{deg}$.

\subsection{Details on the implementation of the RMRAC strategy}

In implementing the control action (5), the following issues have to be considered.

1) The reference model and the adaptive mechanism are discretized using the Tustin method with a sampling time $T_{s}$ of $1 \mathrm{~ms}$.

2) As it usually happens when implementing adaptive strategies, the quantities that modulate the adaptive gains in (7) have to be chosen heuristically as a tradeoff between convergence time and reactivity of the control action. In this work, the parameters $\Gamma$ and $\gamma$ are selected from a finite set so that the mean value of the tracking error is minimized, and they are set as $\Gamma=\operatorname{diag}\{5,5,10,10\}$ and $\gamma=5$.

3) According to [1], to prevent a possible drift of the adaptive gains or equivalently to keep the adaptive gains bounded in presence of noise, the $\sigma$-modification strategy is exploited. As a consequence, (7a) and (7d) are implemented as

$$
\begin{aligned}
& \dot{\psi}=\varepsilon \Gamma \phi-\sigma_{s}(\psi) \Gamma \psi \\
& \dot{\chi}=\gamma \varepsilon \xi-\sigma_{s}(\psi) \gamma \chi
\end{aligned}
$$

where the function $\sigma(\psi)$ is

$$
\sigma_{s}(\psi)=\left\{\begin{array}{lll}
0, & \text { if } & \|\psi\|<M_{0} \\
\left(\frac{\|\psi\|}{M_{0}}-1\right)^{q_{0}} \sigma_{0}, & \text { if } & M_{0} \leqslant\|\psi\| \leqslant 2 M_{0} \\
\sigma_{0}, & \text { if } & \|\psi\|>2 M_{0}
\end{array}\right.
$$

with $M_{0}, q_{0}$ and $\sigma_{0}$ are positive constants. In particular, the threshold $M_{0}$ should be chosen as greater than $\left\|\psi^{\star}\right\|$. Hence, for the applicability of the $\sigma$-modification method, a rough estimation of $\left\|\psi^{\star}\right\|$ is required. On the other hand, this condition is not restrictive, because in many practical scenarios, e.g., when controlling mechanical plants, a rough estimation of $\left\|\psi^{\star}\right\|$ can be derived from the nominal plant parameters, and $M_{0}$ can be always chosen to be big enough to take into account uncertainty on this norm. In this work, we have set the maximum leakage factor $\sigma_{0}$ as $\sigma_{0}=0.1$, $q_{0}=2$ and $M_{0}=10^{2}$.

4) A first-order filter as smooth trajectory reference (STR) is introduced to limit the tracking error during rapid variations of the reference signal.

\section{$6 \quad$ Experimental results}

The RMRAC algorithm has been widely tested over a long reference signal of the throttle position. This reference input is composed by concatenating the signals listed

\begin{tabular}{|c|c|c|c|c|}
\hline Signal & Type & Amplitude (deg) & Bias (deg) & Period (s) \\
\hline 1 & Constant & 20 & & \\
\hline 2 & Square & 25 & 45 & 6 \\
\hline 3 & Sinusoid & 25 & 45 & 6 \\
\hline 4 & Square & 25 & 55 & 6 \\
\hline 5 & Sinusoid & 25 & 45 & 6 \\
\hline 6 & Square & 10 & 50 & 6 \\
\hline 7 & Sinusoid & 10 & 50 & 6 \\
\hline 8 & Constant & 40 & & \\
\hline 9 & Square & 25 & 45 & 6 \\
\hline 10 & Sinusoid & 25 & 45 & 6 \\
\hline 11 & Square & 25 & 55 & 6 \\
\hline 12 & Sinusoid & 25 & 55 & 6 \\
\hline 13 & Square & 10 & 50 & 6 \\
\hline 14 & Sinusoid & 10 & 50 & 6 \\
\hline 15 & Constant & 40 & & \\
\hline 16 & Square & 25 & 55 & 3 \\
\hline 17 & Square & 25 & 55 & 2 \\
\hline 18 & Sinusoid & 25 & 55 & 3 \\
\hline 19 & Sinusoid & 25 & 55 & 1 \\
\hline 20 & Square & 35 & 55 & 5 \\
\hline 21 & Square & 35 & 55 & 3 \\
\hline 22 & Sinusoid & 35 & 55 & 5 \\
\hline 23 & Sinusoid & 35 & 55 & 1 \\
\hline 24 & Square & 7.5 & 52.5 & 5 \\
\hline 25 & Sinusoid & 10 & 50 & 4 \\
\hline 26 & Steps & & & \\
\hline 27 & Sinusoid & 25 & 55 & 2 \\
\hline 28 & Steps & & & \\
\hline 29 & Sinusoid & 6 & 14 & 4 \\
\hline 30 & Sinusoid & 6 & 14 & 2 \\
\hline 31 & Square & 6 & 14 & 4 \\
\hline 32 & Square & 6 & 14 & 3 \\
\hline 33 & Constant & 20 & & \\
\hline
\end{tabular}
in Table 1, and it comprises square, sinusoidal, and step functions with different amplitude, bias, and frequency.

Table 1 Signals that compose the input to the reference model

In particular, signal 26 is a sequence of steps of amplitude $15 \mathrm{deg}$ starting from $15 \mathrm{deg}$ and ranging to a maximum of $90 \mathrm{deg}$ and back. Analogously, signal 28 is a sequence of steps of amplitude $5 \mathrm{deg}$ starting from $10 \mathrm{deg}$ and ranging to a maximum of $90 \mathrm{deg}$ and back.

When the time increases, the control gains evolve, which 
causes a better and better tracking of the reference trajectory as shown in Fig. 4 . In Fig. 4 , the adaptive gains $\psi(t)$ are bounded.

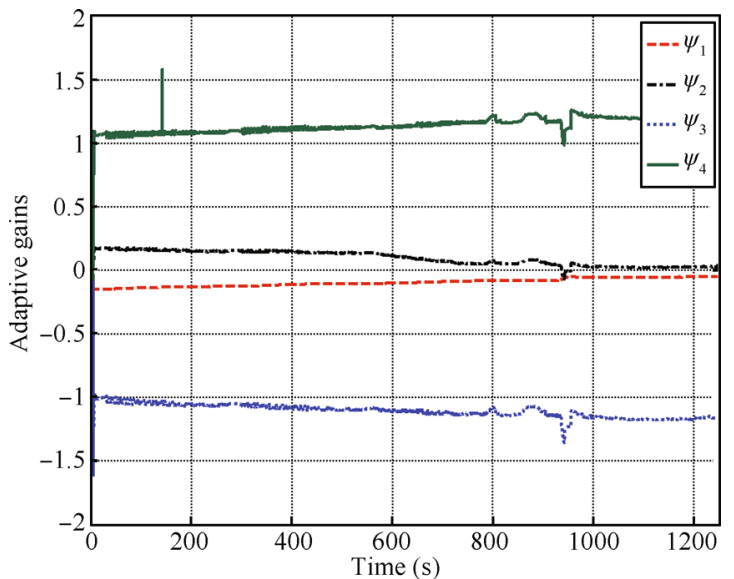

Fig. 4 Experimental adaptive gains over the entire maneuver

We note that the control system does not need to go through a learning phase when this adaptive controller is implemented on a commercial ETB with the adaptive gains starting from zero every time the engine gets restarted. Indeed, the gains could be set to the final values reached in the last engine cycle, before the engine is turned off, and then adapted to cope with uncertainties and external disturbances Moreover, at the start-up of the engine, the throttle reference signals required by the idle-speed controller, which must be tracked during the idle speed regime ${ }^{[16]}$, can be used for starting the updating mechanism of the control gains.

The following paragraphs detail the tracking performance for some reference signals of automotive interest. Furthermore, we denote $e=\theta_{m}-\theta_{t h}$ as the tracking error between the demanded reference plate position provided by the reference model $\theta_{m}$, and the experimental plate position $\theta_{t h}$.

1) Square wave variation of the throttle plate position. Figs. 5 and 6 show the tracking performance in the case of different square wave reference signals. In particular, Fig. 5 (a) shows the experimental results for wide opening and closing maneuvers where the valve should be opened and closed over a position range of about $70 \mathrm{deg}$. Over this range of angular positions, the spring characteristic changes And as a consequence, the disturbance term acting on the plant dynamics is not a constant that can be compensated with classical integral control actions. Nevertheless, the adaptive scheme is robust with respect to this nonlinear disturbance and provides a tracking error that never exceeds 7 deg, as clearly shown in Fig. 5 (b). Furthermore, as apparent from Fig. 5 (a), the plate can be driven close to the end stroke of the valve without impacting. Hence, the lifespan of the component increases. The corresponding control signal is always within the feasible range, as shown in Fig. 5 (c).

Similar tracking results are achieved for the entire set of square waves in Table 1. For the sake of completeness, the case of a square wave reference input with amplitude $25 \mathrm{deg}$, bias $55 \mathrm{deg}$, and period $2 \mathrm{~s}$ is reported in Figs. 6 (a), (b), and (c).

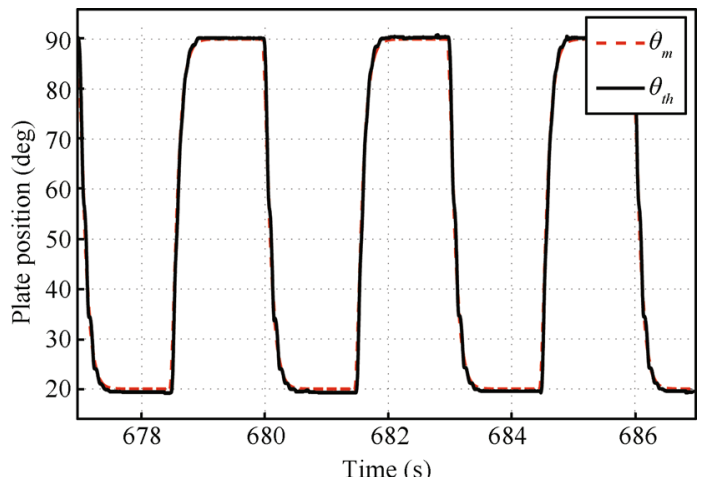

(a)

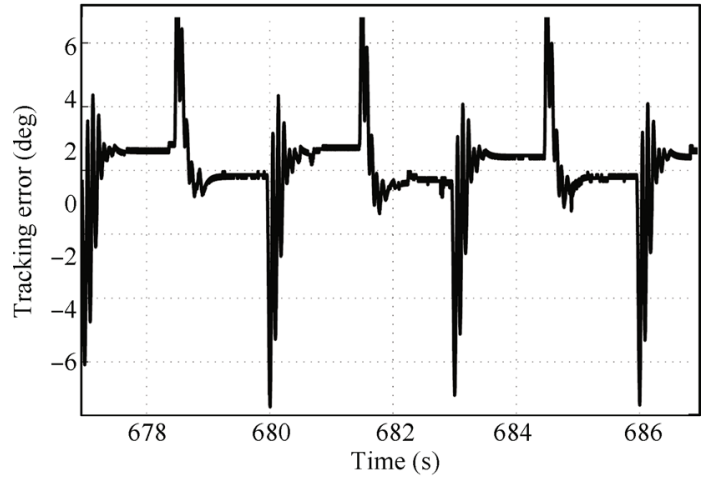

(b)

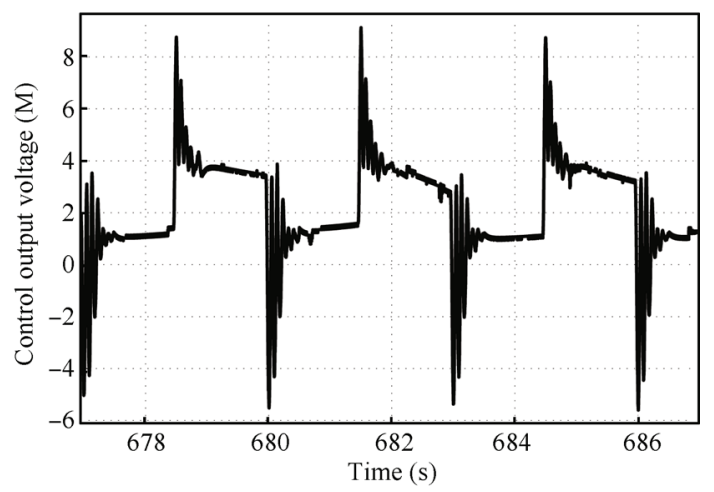

(c)

Fig. 5 Experimental results for a square wave with an amplitude of $35 \mathrm{deg}$, a bias of $55 \mathrm{deg}$, and a period of $3 \mathrm{~s}$. (a) Angular plate position $\theta_{m}$ reference plate trajectory (dashed line), and plate position $\theta_{t h}$ (solid line); (b) Tracking error; (c) Controller output voltage

2) Sinusoidal wave variation of the throttle plate position. We now analyze the closed-loop response in the presence of sinusoidal maneuvers. The complexity here is to prevent the valve from getting stuck due to friction when the plate velocity changes the sign. As shown in Figs. 7 and 8, unwanted stick and slip phenomena that affect the open-loop dynamics (see Fig. 3 (a) are not present in the closed-loop behavior). For both cases considered in Figs. 7 and 8, the tracking error is below $2.5 \mathrm{deg}$.

3) Sequence of rising step variations of the throttle plate position. Fig. 9 shows the tracking performance when the input command is a sequence of rising step from $40 \mathrm{deg}$ to $90 \mathrm{deg}$. The closed-loop response closely matches the model 
reference dynamics. The maximum transient error is $4 \mathrm{deg}$, and it becomes smaller than $0.3 \mathrm{deg}$ in steady-state.

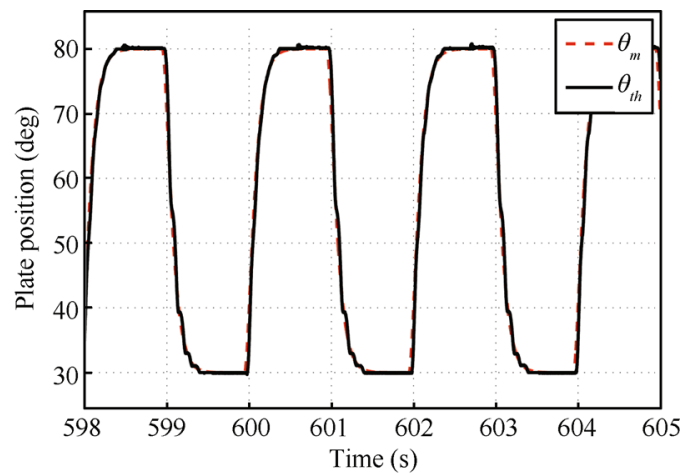

(a)

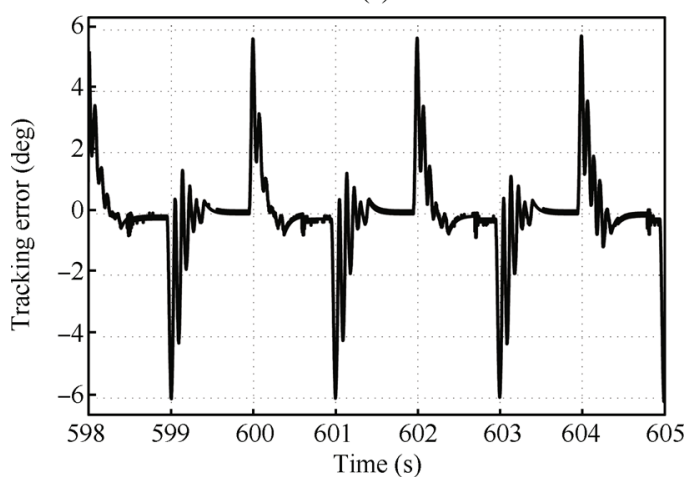

(b)

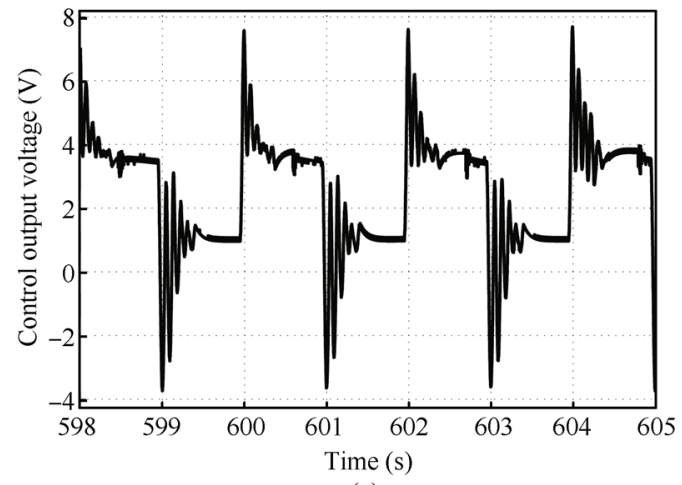

(c)

Fig. 6 Experimental results for square wave with an amplitude of $25 \mathrm{deg}$, a bias of $55 \mathrm{deg}$, and a period of $2 \mathrm{~s}$. (a) Angular plate position, $\theta_{m}$, reference plate trajectory (dashed line), and $\theta_{t h}$, plate position (solid line); (b) Tracking error; (c) Controller output voltage

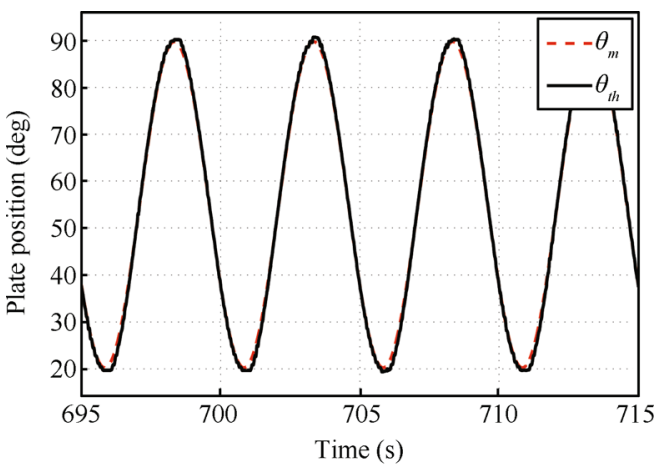

(a)

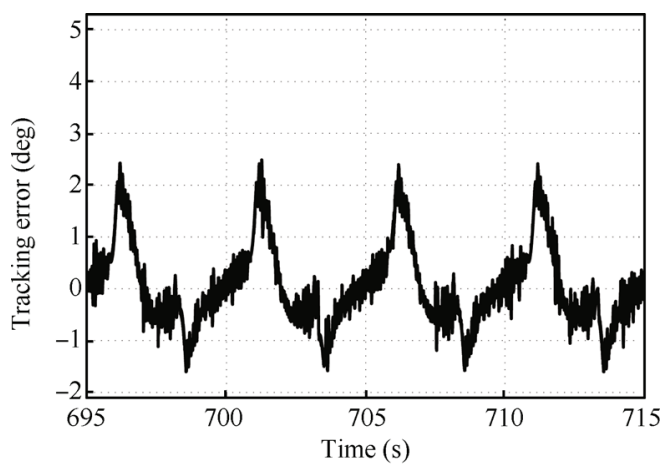

(b)

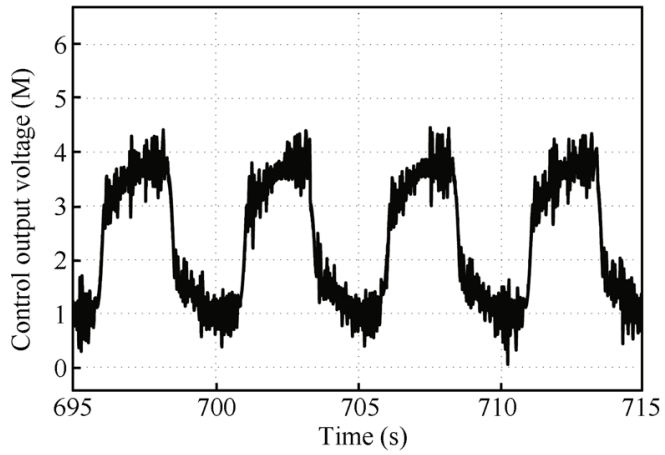

(c)

Fig. 7 Experimental results for sinusoidal throttle opening with an amplitude of $35 \mathrm{deg}$, a bias of $55 \mathrm{deg}$, and a period of $6 \mathrm{~s}$. (a) Angular plate position $\theta_{m}$, reference plate trajectory (dashed line), and plate position $\theta_{t h}$ (solid line); (b) Tracking error; (c) Controller output voltage

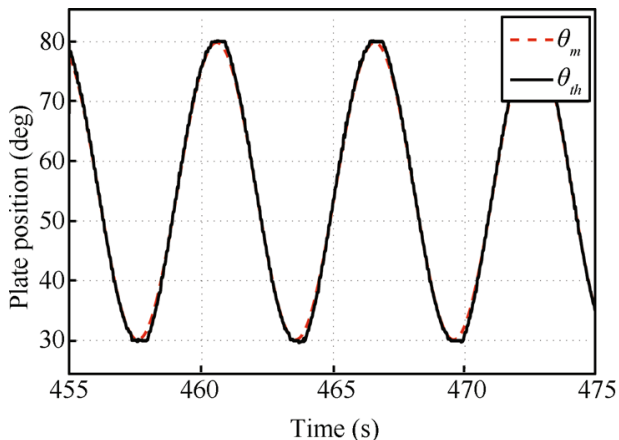

(a)

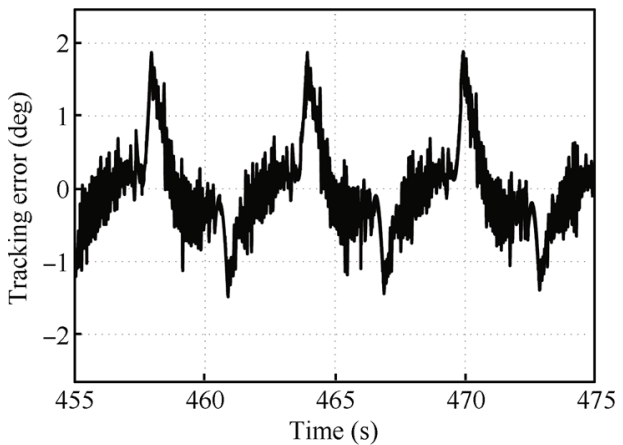

(b) 


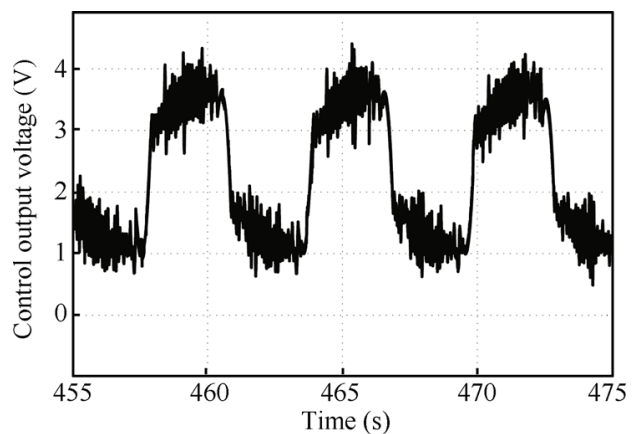

(c)

Fig. 8 Experimental results for sinusoidal throttle opening with an amplitude of $25 \mathrm{deg}$, a bias of $55 \mathrm{deg}$, and a period of $6 \mathrm{~s}$. (a) Angular plate position $\theta_{m}$, reference plate trajectory (dashed line), and plate position (solid line $\theta_{t h}$ ); (b) Tracking error; (c) Controller output voltage

4) Small amplitude step variations of the throttle plate position. Another significant test is the case of small amplitude reference signals when the presence of stick-slip motion due to friction becomes particularly relevant because position and velocity variations are relatively low ${ }^{[13]}$. Here, we consider at first the case of a sequence of small steps starting from $45 \mathrm{deg}$ to $25 \mathrm{deg}$ selected from signal 28 in Table 1. As shown in Fig. 10, the robust adaptive controller copes well with such unwanted nonlinear perturbations. To persistently excite the plant nonlinearities due to friction, which again is not taken explicitly into account during the control system design, we select as reference input a square

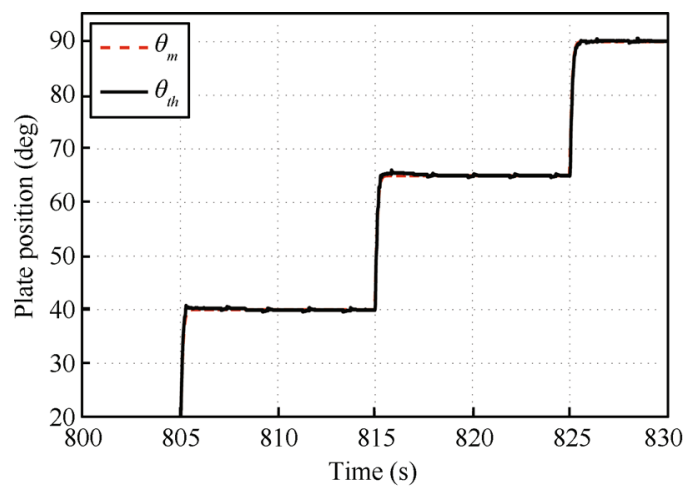

(a)

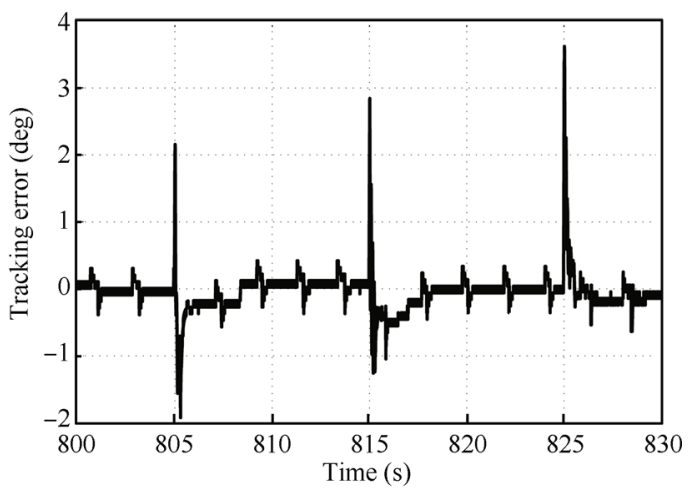

(b)

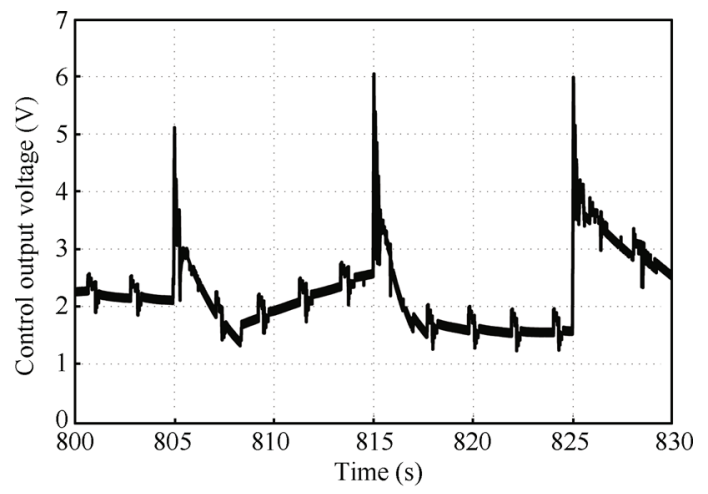

(c)

Fig. 9 Experimental results for a sequence of rising steps: (a) Angular plate position, $\theta_{m}$, reference plate trajectory (dashed line), and $\theta_{t h}$, plate position (solid line); (b) Tracking error; (c) Controller output voltage

wave with a small amplitude. Fig. 11 shows the experimental results for this operating condition. We remark that despite the presence of nonlinear disturbance and unmodeled dynamics that are not negligible, tracking performance does not deteriorate.

Finally, we point out that not only the adaptive gains $\psi(t)$, are bounded as shown in Fig. 4, but also all the closed-loop signals computed by the controller are bounded as guaranteed by Theorem 2. As shown in Fig. 12, the $\chi$-term, which is computed via the nonlinear differential equation (7d), is bounded. The boundedness of the other closed-loop signals can be derived from the boundedness of

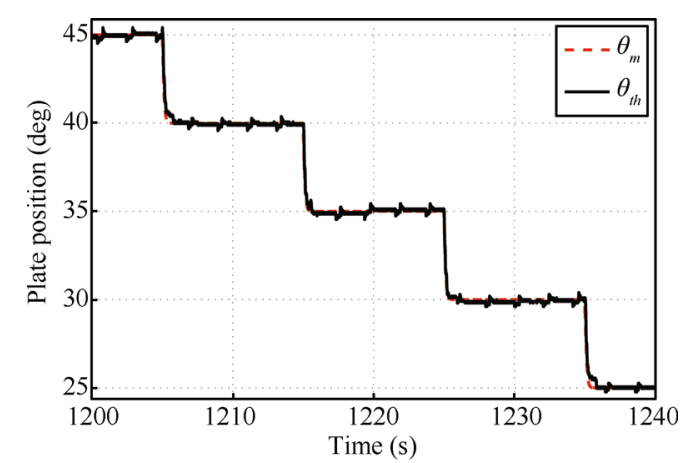

(a)

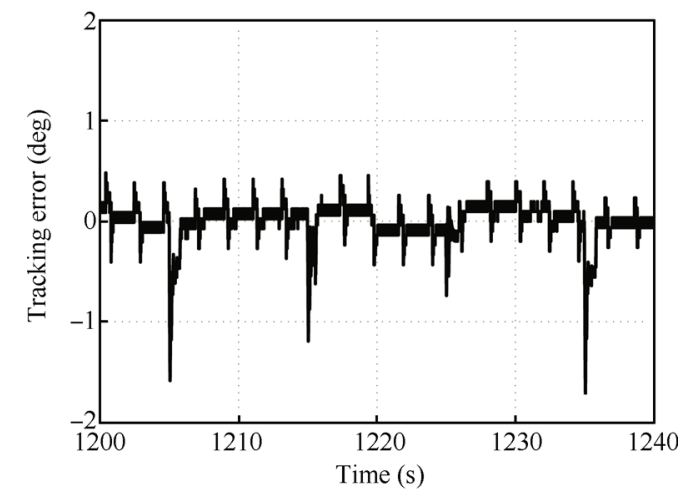

(b) 


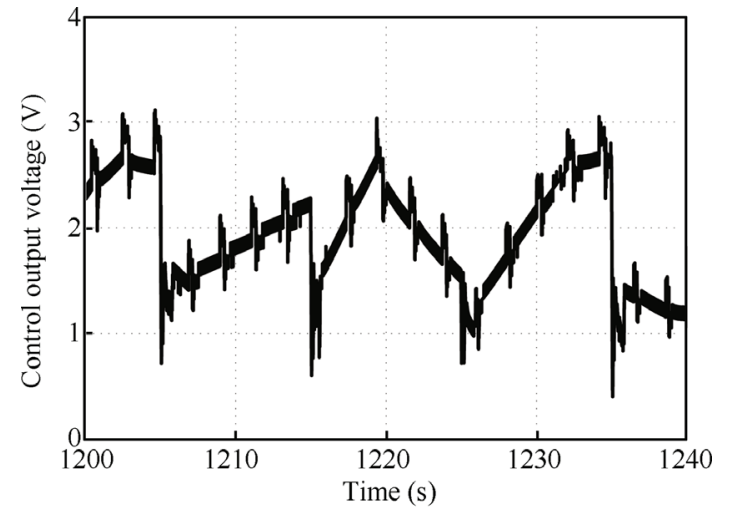

(c)

Fig. 10 Experimental results for a sequence of falling steps. (a) Angular plate position, $\theta_{m}$, reference plate trajectory (dashed line), and $\theta_{t h}$, plate position (solid line); (b) Tracking error; (c) Controller output voltage

the throttle position, i.e., $y=\theta_{t h}$ and that of the control signal $u$, the boundedness of which has been experimentally verified. $\tau_{1}, \tau_{2}, \phi, u_{f}$ and $n_{d}$, defined in (6b) (6c), (7c), and (7f) are bounded, as they are the outputs of asymptotically stable linear time invariant systems excited by bounded signals, while the closed-loop signals $m_{s}, \varepsilon$ and $\xi$ are bounded as they are static function of bounded variables.

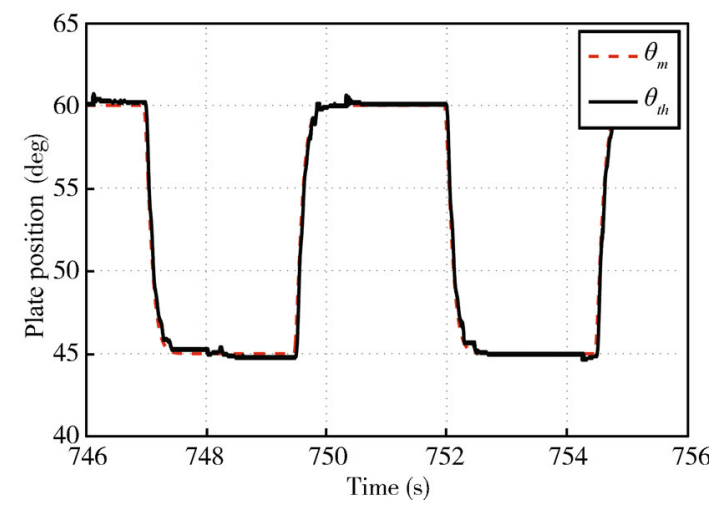

(a)

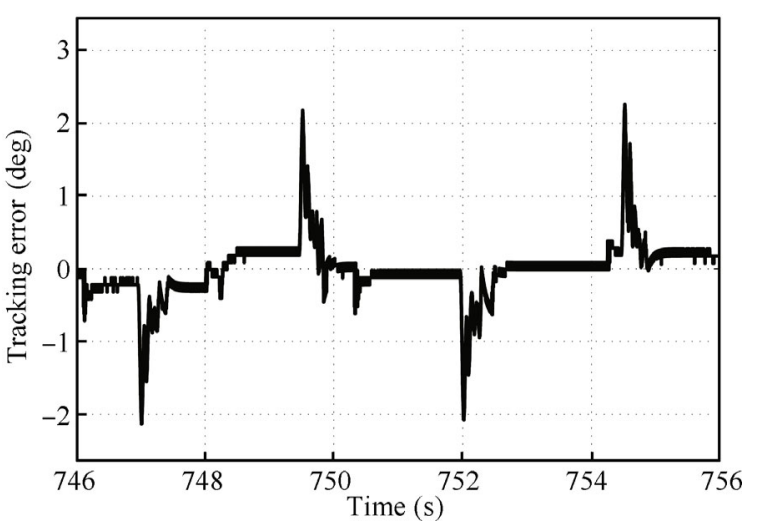

(b)

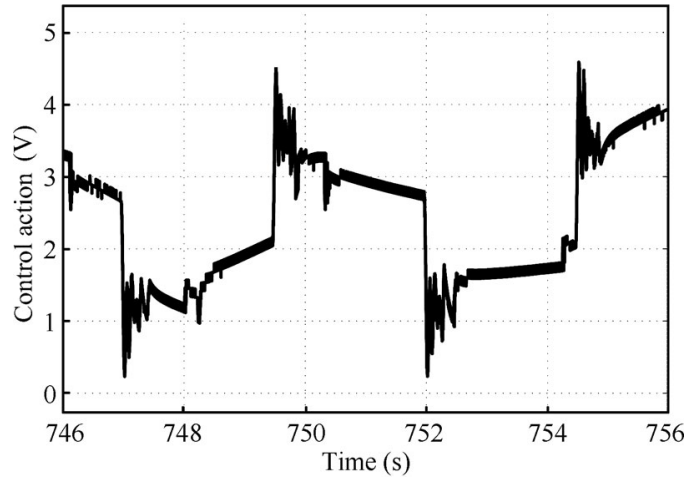

(c)

Fig. 11 Experimental results for small square wave with an amplitude of $6 \mathrm{deg}$, a bias of $14 \mathrm{deg}$ and a period of $4 \mathrm{~s}$. (a) Angular plate position, $\theta_{m}$, reference plate trajectory (dashed line), and $\theta_{t h}$, plate position (solid line); (b) Tracking error; (c) Controller output voltage

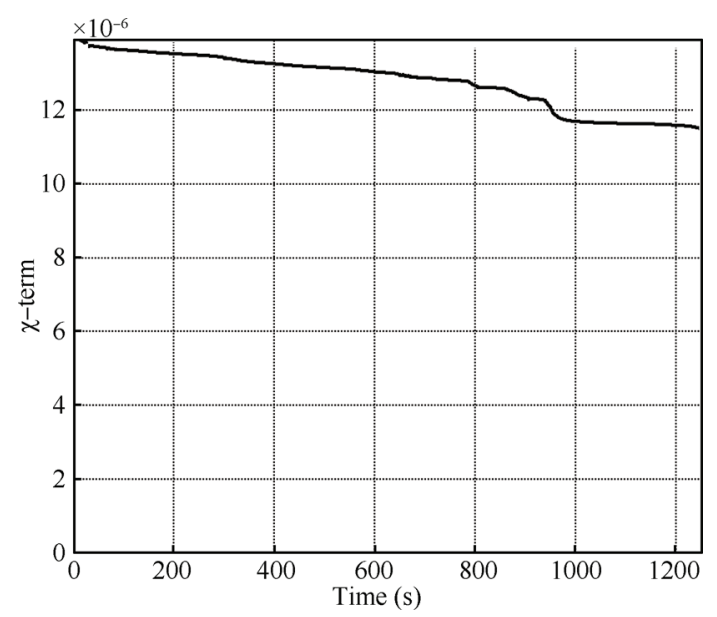

Fig. 12 Experimental $\chi$-term over the entire maneuver

\section{Experimental comparison between RMRAC and model-based control}

In this section, to confirm the effectiveness of adaptive control methods in controlling the complex dynamics of this automotive testbed actuator, we carry out an extensive experimental comparison of the closed-loop performance provided by the RMRAC strategy and that given by a model-based controller with fixed gains. This controller, referred to as the PINLFF proportional integral nonlinear feedforward (PINLFF) is composed of a model-based nonlinear feedforward compensator for plant nonlinearities and a PI feedback controller to compensate parameter mismatch and disturbances. Further details on the design of a modelbased controller can be found in [26]. Here we just stress that this classical control strategy requires, for the tuning of its control gains, a precise identification of plant parameters, because its closed-loop performance strongly depends on the accuracy of the identification results.

For the sake of a fair comparison, the same reference model, sample time, and reference input are used for both controllers. Furthermore, to visit all the state space regions 
where the plant is strongly nonlinear (i.e., the region around the limp-home position, zero plate velocity as well as positions close to the end-stroke of the valve), the reference signal is again the long maneuver composed by the signals listed in Table 1.

In what follows closed-loop performance is analyzed by using as performance indexes: 1) the mean value of the absolute tracking error $(34 a) ; 2)$ its standard deviation (34b); 3) the maximum absolute tracking error (34c); and 4$)$ the average control effort (34d). In more detail, we compute

$$
\begin{aligned}
\mu & =\frac{1}{T} \int_{0}^{T}|e(t)| \mathrm{d} t \\
\sigma & =\sqrt{\mu\left([|e(t)|-\mu(e)]^{2}\right)} \\
\kappa & =\max _{t \in[0, T]}|e(t)| \\
P & =\frac{1}{T} \int_{0}^{T} \frac{v_{a}(t)^{2}}{R} \mathrm{~d} t
\end{aligned}
$$

where $T$ is the time interval of observation, the tracking error $e$ is defined in Section 6, and $P$ is the mean control effort. (Note that $P$ is related to the mean electrical power, provided by the battery, due to the losses in the armature coil when current $i \approx \frac{v_{a}}{R}$.)

\subsection{Tracking performance comparison}

To compare the tracking capabilities of the adaptive algorithm with respect to those given by the model-based strategy, we split the reference signal in Table 1 in the following relevant subsets.

1) Learning, from signals 2 to 8 . After this first set of maneuvers, the transient of the adaptive gains due to the activation of the adaptive controller is supposed to be extinguished. The tracking error over this set is used for starting up the adaptation mechanism since the adaptive gains are initially set to zero.

2) After learning, from signals 9 to 15 . The sequence of signals composing this set is the same as that of learning-set. If the adaptive controller works properly, the adaptive gains should not vary too much from the values reached at the end of the first set, since they should be entered definitively in their residual set. Furthermore, if the transient of the adaptive gains is extinguished over the first set, then the tracking error is not expected to improve further on the same set of reference signals.

3) Miscellaneous signals, from signals 16 to 25 . This set collects different signals whose bias and amplitude mimic those required when gasoline engines work at medium and high loads. Hence, these are typical signals that have to be tracked during the normal engine operations.

4) Small signal, from signals 29 to 32 . This set contains signals with small amplitude oscillations around the limphome position where the dynamics of the plant are strongly nonlinear due to the limp-home and friction effects (see Section 4). This set contains typical signals that have to be tracked to implement idle speed control strategies ${ }^{[16]}$ that use ETB as an actuator. In so doing the traditional bypass valves can be removed.
5) Sequence of steps, signals 26 and 28 . This set is composed of a sequence of steps included in the long maneuver. Hence, its study allows an analysis of the closed-loop performance for different steady state regimes.

6) Square waves. This set collects all the square waves of the long maneuver (except those belonging to the learningset) with different periods, amplitudes and bias. It is used to test closed-loop performance in the presence of sudden tip-in/tip-out maneuvers. These maneuvers occur when the driver demands rapid increasing/decreasing of the engine thrust.

7) Sinusoidal waves. This set contains all the sinusoidal waves of the entire input signal (except those belonging to the learning-set), and it is used to test closed-loop performance in presence of smooth tip-in/tip-out maneuvers.

For each set, all the performance indexes have been computed on experimental data both for the adaptive strategy and the model-based controller. The results reported in Figs. 13 and 14 clearly show that the adaptive control method provides better tracking performance. In particular, we note the following:

1) The transient of the adaptive gains due to the activation of the adaptive controller is short since the performance indexes computed on the first set (learning-set) are comparable with those obtained analyzing the second set (after learning-set).

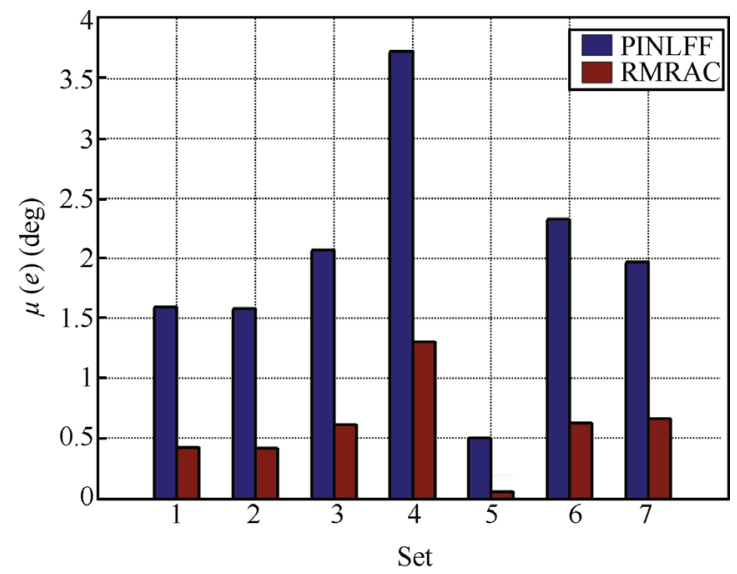

(a)

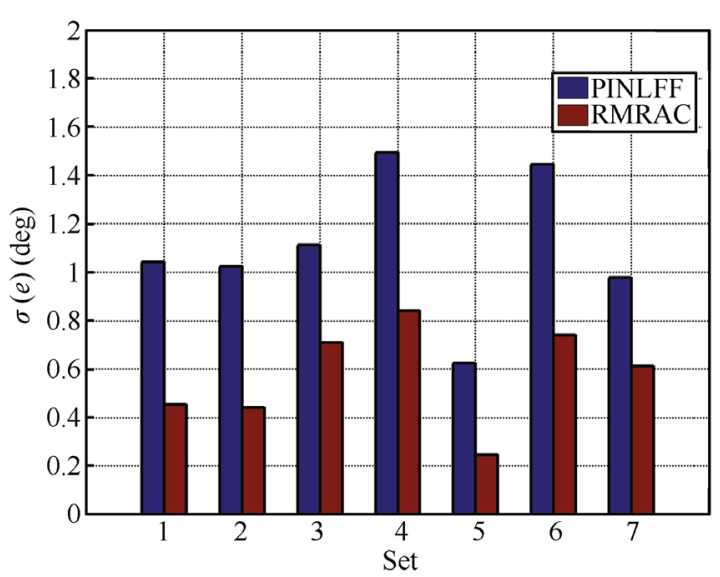

(b) 


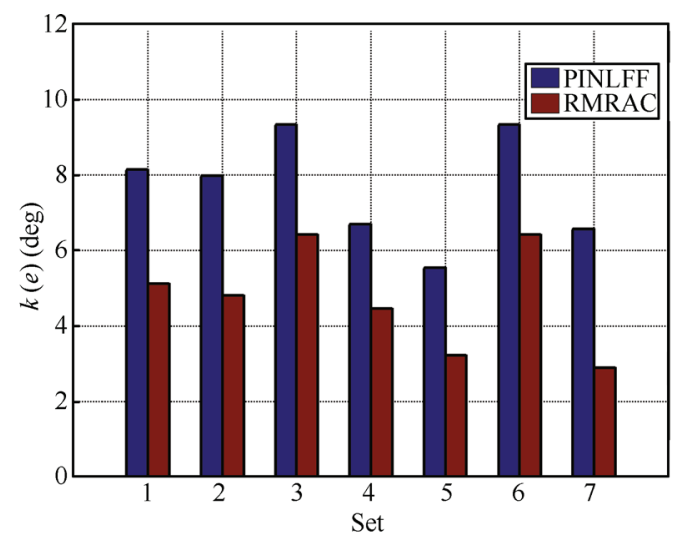

(c)

Fig. 13 Experimental results. Performance indexes for each set: (a) $\mu$, mean value of the absolute tracking error; (b) $\sigma$, standard deviation of the absolute tracking error; (c) $\kappa$, maximum absolute tracking error

2) The mean value of the tracking error provided by the adaptive algorithm never exceeds $1.2 \mathrm{deg}$ with a maximum standard deviation of about $0.8 \mathrm{deg}$, as clearly shown in the Figs. 13 (a) and (b), respectively. Instead, even if a detailed model of the ETB system and an accurate plant parameter identification are used for the design of the PINLFF strategy, the achieved maximum mean value error is about $4 \mathrm{deg}$ with a standard deviation of $1.5 \mathrm{deg}$. Hence, the adaptive method improves the tracking performance of about $70 \%$ and $45 \%$ with respect to the mean value error and the standard deviation, respectively.

3) As it is apparent from Fig. 13 (c), the RMRAC algorithm tracks small signals around the limp-home position (set 4) much better than the model-based strategy does.

4) The closed-loop performance of the adaptive method is superior with respect to that of the model-based strategy not only in terms of tracking of the reference trajectory but also with respect to the control effort, as shown in Fig. 14 . Indeed, a reduction of mean electrical power is obtained over all the sets except for set 4, where an increased control effort was expected to tame the discontinuous nonlinearities characterizing the limp-home zone.

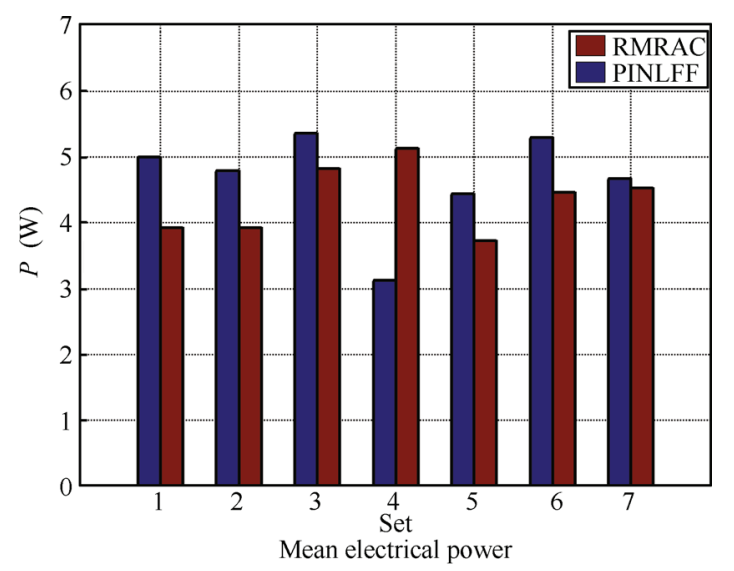

Fig. 14 Experimental results. $P$, mean electrical power of the control action for each set

\subsection{Regulation performance comparison}

We now test the regulating capabilities for both control strategies by evaluating the performance indices $\mu, \sigma$, and $\kappa$ in steady state regime for a wide range of constant input reference commands. Fig. 15 reports the experimental results, which show the performance indices computed when the set point $r$ belongs to $\{10+5 k \mathrm{deg}, k=0,1, \cdots, 16\}$. Again, the adaptive strategy provides better performance when compared with the PINLFF controller. More in detail:

1) Even though both control strategies provide a comparable standard deviation of the absolute tracking error as shown in Fig. 15 (b), the mean value tracking error is much smaller when the adaptive strategy is used for steering plant dynamics. In particular, as shown in Fig. 15 (a), the $\mu$-index is always below $0.2 \mathrm{deg}$. On the other hand the model-based controller provides a $\mu$-index of $1.2 \mathrm{deg}$ and $1.7 \mathrm{deg}$ for reference set points around the limp-home position $(r=10 \mathrm{deg}$ and $r=15 \mathrm{deg})$ where the RMRAC gives a mean value regulating error always less than $0.06 \mathrm{deg}$.

2) The maximum tracking error computed when the adaptive controller is inserted in the loop is smaller than that provided by the model-based strategy (see Fig. 15 (c)). Only at $90 \mathrm{deg}$ is the $\kappa(e)$-index slightly greater in the case of the adaptive algorithm. On the other hand, for set points close to the limp-home position, the regulating performance provided by the RMRAC is superior with respect to that given by the PINLFF controller.

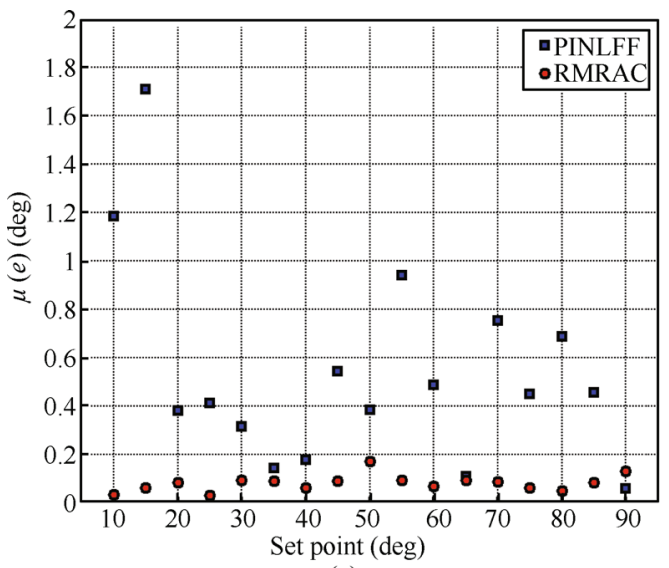

(a)

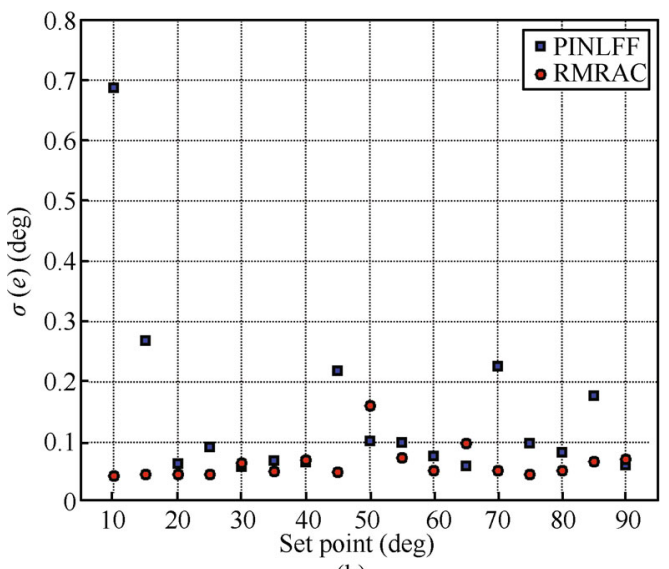

(b) 


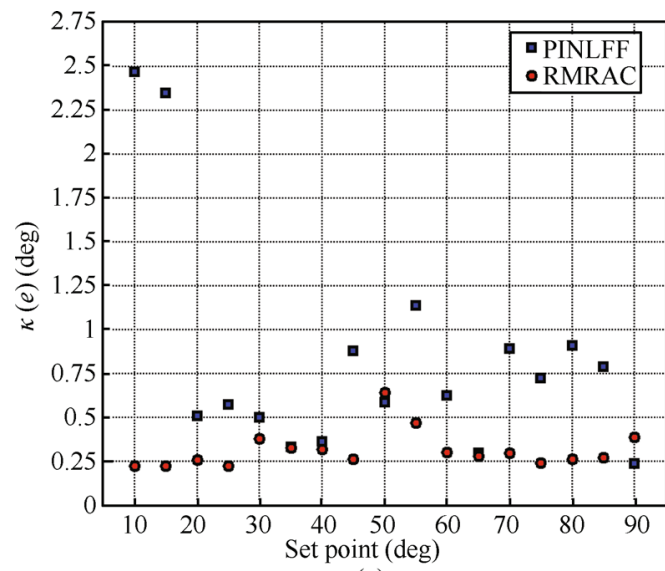

(c)

Fig. 15 Performance indices computed in steady state.

Mean value of the absolute tracking error, $\mu(e)$; (b) Standard deviation of the absolute tracking error, $\sigma(e)$; (c) Maximum absolute tracking error, $\kappa(e)$

\section{Conclusions}

In this paper, a robust output model reference adaptive control has been designed to show its applicability to mechatronic automotive devices. The ETB system was chosen as the experimental testbed system, as its nonlinearities and model uncertainties produce typical unwanted phenomena encountered in automotive electromechanical subsystems. Unlike other adaptive control methods already used for this actuator, the control approach used here allows estimation of the residual tracking error at the design time. Experimental results for reference signals of automotive interest and an in-depth comparison with a model-based control strategy clearly show that the adaptive controller is a viable and simple solution to tame nonlinearities, such as nonlinear frictions, variable restoring torque, and deadzones, which are common to many mechatronic automotive devices.

\section{Appendix: Experimental setup}

The experimental setup is the same used in $[10,13]$ and it consists of: 1) an electronic throttle body (DV-E5, Bosch), (see Fig. 16), embedding a DC motor; 2) two dual resistive angular position sensors; 3) a battery voltage sensor; 4) an $h$-bridge power circuit (to drive the DC motor); 5) a hall effect current sensor (LTA 50P/SP1, LEM); 6) signal conditioning circuits; 7) a station for Rapid Control Prototyping $(\mathrm{RCP})^{[13]}$

The open-loop response of the ETB plant can be summarized through two characteristic times, namely, the time necessary to open widely the valve under a battery voltage step $\left(V_{\text {bat }} \approx 12 \mathrm{~V}\right), t_{\text {open }} \approx 100 \mathrm{~ms}$, and the current-less return time, that is, the time necessary to close the valve in free evolution, $t_{c l} \approx 350 \mathrm{~ms}^{[10]}$.

The RCP is a dspace based multiprocessor system equipped with the DS1003 (DSP TMS320C40, 60 Mflops) and DS1004 (DEC Alpha AXP 21164, 600 Mflops) processor boards. An analog DS2201 (20 Channels, 12 bit, $30 \mathrm{kHz}$ ) and a digital DS4002 (8 Channels, capture/compare resolution $30 \mathrm{bit} / 200 \mathrm{~ns}$, frequency $\max 833 \mathrm{kHz}$ ) board allow the
I/O handling. The DSP is programmed in Matlab/Simulink environment, and the experiments are managed and instrumented by a controldesk application (dSpace). Furthermore, an oscilloscope (TDS-3014, Tektronix) is used to perform high-frequency measurements ${ }^{[13]}$.

Note that, the execution of our control task, using the hardware described above, is performed in $10 \mu \mathrm{s}$ with a sample period of $1 \mathrm{~ms}$.

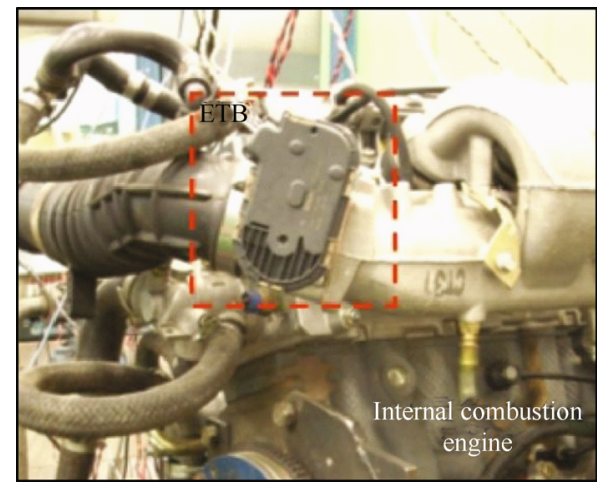

Fig. 16 Experimental ETB device

\section{References}

[1] P. Ioannou, B. Fidan. Adaptive Control Tutorial, Philadelphia: SIAM, 2006.

[2] P. A. Ioannou, J. Sun. Robust Adaptive Control, Upper Saddle River, NJ: Prentice Hall, 1996.

[3] M. Krstic, I. Kanellakopoulos, P. V. Kokotovic. Nonlinear and Adaptive Control Design, New York: Wiley, 1995.

[4] K. J. Astrom, B. Wittenmark. Adaptive Control, 2nd ed., Reading, Massachusetts: Addison Wesley, 1994.

[5] Y. D. Landau. Adaptive Control: The Model Reference Approach, New York: Marcel Dekker Incorporated, 1979.

[6] J. K. Astrom. A survey of adaptive control applications. In Proceedings of the 34th IEEE Conference on Decision and Control, IEEE, New Orleans, LA, USA pp. 649-654, 1995.

[7] B. Wittenmark. A survey of adaptive control applications. In Proceedings of IEEE Industry Applications Society Dynamic Modeling Control Applications for Industry Workshop, IEEE, Vancouver, BC, USA, pp. 32-36, 1997.

[8] K. J. Astrom. Theory and applications of adaptive controlAsurvey. Automatica, vol. 19, no. 5, pp. 471-486, 1983.

[9] M. Boukattaya, T. Damak, M. Jallouli. Robust adaptive control for mobile manipulators. International Journal of Automation and Computing, vol. 8, no. 1, pp. 8-13, 2011.

[10] M. Di Bernardo, A. Di Gaeta, U. Montanaro, S. Santini. Synthesis and experimental validation of the novel LQNEMCSI adaptive strategy on an electronic throttle valve. IEEE Transactions on Control System Technology, vol.18, no. 6, pp. 1325-1337, 2010

[11] M. Di Bernardo, A. Di Gaeta, V. Giglio, U. Montanaro, $\mathrm{S}$. Santini. Design and validation of a novel model reference adaptive algorithm to control ETB for drive-bywire applications. International Journal of Passenger CarsMechanical Systems, vol. 2, no. 1, pp. 1268-1284, 2009. 
[12] M. Di Bernardo, A. Di Gaeta, U. Montanaro, J. M. Olm, S. Santini. Discrete-time MRAC with minimal controller synthesis of an electronic throttle body. In Proceedings of the 18th IFAC World Congress, IFAC, Universitá Cattolica del Sacro Cuore, Milano, Italy, pp. 5064-5069, 2011.

[13] M. Di Bernardo, A. Di Gaeta, U. Montanaro, J. M. Olm, S. Santini. Experimental validation of the discrete-time MCS adaptive strategy. Control Engineering Practice, vol.21, no. 6, pp. 847-859, 2013.

[14] Q. Z. Gao, X.-J. Xie. Robustness analysis of discrete-time indirect model reference adaptive control with normalized adaptive laws. International Journal of Automation and Computing, vol. 7, no. 3, pp. 381-388, 2010.

[15] A. Di Gaeta, G. Fiengo, A. Palladino, V. Giglio. Design and experimental validation of a model-based injection pressure controller in a common rail system for GDI engine. In Proceedings of American Control Conference, IEEE, San Francisco, CA, USA pp. 5273-5278, 2011.

[16] A. Di Gaeta, U. Montanaro, V. Giglio. Idle Speed Control of GDI-SI Engines via ECU-1D Engine Co-simulation, SAE Technical Paper (2010), no. 2010-01-2220.

[17] C. Rossi, A. Tilli, A. Tonielli. Robust control of a throttle body for drive by wire operation of automotive engines. IEEE Transactions on Control Systems Technology, vol. 8, no. 6, pp. 993-1002, 2000.

[18] M. S. Sangha, D. L. Yu, J. B. Gomm. Robustness assessment and adaptive FDI for car engine. International Journal of Automation and Computing, vol. 5, no. 2, pp. 109-118, 2008.

[19] D. Pavković, J. Deur, M. Jansz, N. Perić. Adaptive control of automotive electronic throttle. Control Engineering Practice, vol. 14, no. 2, pp. 121-136, 2006.

[20] J. Deur, D. Pavkovic, N. Peric, M. Janz. An electronic throttle control strategy including compensation of friction and limp-home effects. In Proceedings of IEEE International Electric Machines and Drives Conference, IEEE, Madison, Wisconsin, USA, pp. 200-206, 2003.

[21] C. C. De Witt, H. Olsson, K. J. Astrom, P. Lischinsky. A new model for control of systems with friction. IEEE Transactions on Automatic Control, vol. 40, no. 3, pp. 419425, 1995.

[22] Y. D. Pan, U. Ozguner, O. H. Dagci. Variable-structure control of electronic throttle valve. IEEE Transactions on Industrial Electronics, vol. 55, no. 11, pp. 3899-3907, 2008.
[23] M. Vašak, M. Baotić, M. Morari, I. Petrović, N. Perić. Constrained optimal control of an electronic throttle. International Journal of Control, vol. 79, no. 5, pp. 465-478, 2006.

[24] M. Vasak, M. Baotic, I. Petrović, N. Peric. Hybrid theory-based time-optimal control of an electronic throttle. IEEE Transactions on Industrial Electronics, vol. 54, no. 3, pp. 1483-1494, 2007.

[25] M. Vasak, M. Baotic, I. Petrovic, N. Peric. Electronic throttle state estimation and hybrid theory based optimal control In Proceedings of IEEE International Symposium on Industrial Electronics, IEEE, Ajaccio, France, vol. 1, pp. 323-328, 2004.

[26] M. di Bernardo, A. di Gaeta, U. Montanaro, S. Santini. A comparative study of the new LQ-MCS control on an automotive electro-mechanical system. In Proceedings of IEEE International Symposium on Circuits and Systems, IEEE, Seattle, WA, USA, pp. 552-555, 2008.

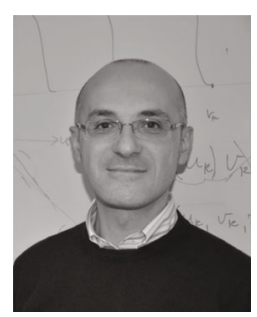

Alessandro di Gaeta received his "Laurea" degree (M. Sc.) cum laude in computer science engineering and his Ph. D. degree in automatic control from the University of Naples "Federico II", Italy in 1999 and 2003, respectively. From 2003 to 2006 he was a postdoctoral research fellow at the Istituto Motori, Italian National Research Council, Italy. Since 2007 he worked as researcher in the automotive control field. He is co-author of the book Common Rail System for GDI Engines as Modelling, Identification, and Control, published by Springer London, January, 2013.

His research interests include modelling, analysis and control of automotive mechatronic systems and of spark ignition engines. E-mail: a.digaeta@im.cnr.it

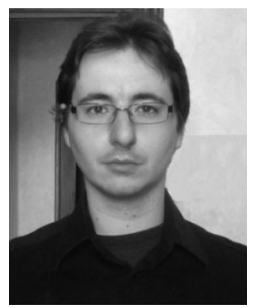

Umberto Montanaro received his "Laurea" degree (M. Sc.) in computer science engineering cum laude (first class with honors) and his Ph. D. in automatic control from the University of Naples Federico II, Italy in 2005 and 2009 , respectively. He is currently a postdoctoral research fellow at the Istituto Motori of the Italian National Research Council. He is also temporary lecturer in automation and process control at the University of Naples Federico II, Italy.

His research interests include automotive control, modeling and control of nonlinear dynamical systems, adaptive control and control of piecewise affine systems.

E-mail: umberto.montanaro@gmail.com, u.montanaro@ im.cnr.it (Corresponding author) 